\title{
Classes de Gevrey em Estruturas Hipo-Analíticas
}

\author{
Paulo Antonio Silvani Caetano
}

\author{
TESE APRESENTADA \\ $\mathrm{AO}$ \\ INSTITUTO DE MATEMÁTICA E ESTATÍSTICA \\ DA \\ UNIVERSIDADE DE SÃO PAULO \\ PARA \\ OBTENÇÃO DO GRAU DE DOUTOR \\ EM \\ MATEMÁTICA
}

\author{
Área de Concentração: Matemática \\ Orientador: Prof. Dr. Paulo Domingos Cordaro
}

Durante a elaboração deste trabalho o autor recebeu apoio financeito da CAPES

- São Paulo, agosto de 2000 - 


\title{
Classes de Gevrey em Estruturas Hipo-Analíticas
}

\author{
Este exemplar corresponde à redação \\ final da tese devidamente corrigida e defendida \\ por Paulo Antonio Silvani Caetano \\ e aprovada pela comissão julgadora.
}

São Paulo, 20 de setembro de 2000.

Banca examinadora:

- Prof. Dr. Paulo Domingos Cordaro (orientador) (IME-USP)

- Prof. Dr. Alfredo Jorge Aragona Vallejo (IME-USP)

- Prof. Dr. Jorge Guillermo Hounie (DM-UFSCar)

- Prof. Dr. Adalberto Panobianco Bergamasco (DM-UFSCar)

- Prof. Dr. Joaquim Tavares (DMat-UFPE) 


\section{Índice}

Introdução 1

1. Hipo-analiticidade Gevrey em codimensão nula ....5

1.1 Estruturas hipo-analíticas com codimensão nula: a base $M \ldots \ldots .5$

1.2 Diferenciabilidade Gevrey segundo $M \ldots \ldots \ldots \ldots \ldots \ldots$

1.3 Exemplos de funções Gevrey segundo $M \ldots \ldots \ldots \ldots \ldots \ldots \ldots$

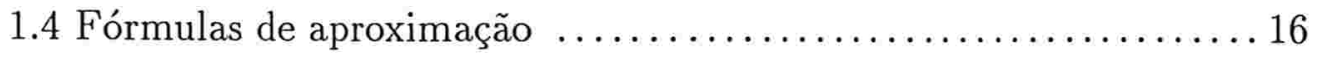

1.5 Uma certa classe de operadores Gevrey segundo M ........... 18

2. Soluções Gevrey hipo-analíticas .................27

2.1 Estruturas hipo-analíticas com codimensão não nula:

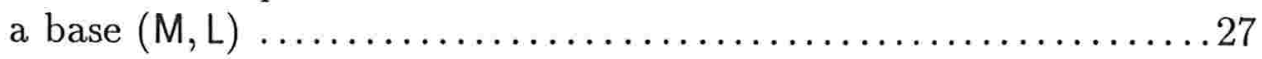

2.2 Diferenciabilidade Gevrey segundo $(\mathrm{M}, \mathrm{L}) \ldots \ldots \ldots \ldots \ldots \ldots \ldots . \ldots . \ldots . \ldots . \ldots 29$

2.3 Funções Gevrey hipo-analíticas ......................... 30

$2.4 \mathrm{O}$ Teorema de Baouendi-Treves Gevrey hipo-analítico ......... 35

3. Resolubilidade Gevrey hipo-analítica parcial ..... 38

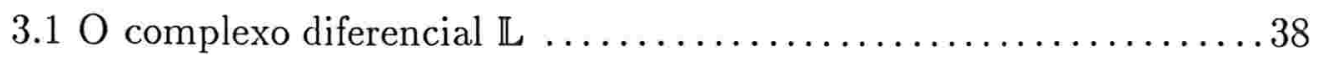

3.2 A resolubilidade local Gevrey hipo-analítica parcial ............ 39

3.3 Resolubilidade $C^{\infty}$ versus Resolubilidade Gevrey hipo-analítica .. 42

4. Resolubilidade Gevrey para funções $(q=1) \ldots \ldots \ldots 46$

4.1 Resolubilidade local em funções Gevrey segundo $(\mathrm{M}, \mathrm{L}) \ldots \ldots \ldots 46$

4.2 A condição $(\mathcal{P})$ versus resolubilidade Gevrey $\ldots \ldots \ldots \ldots \ldots \ldots$

5. Observações Finais .............................. 51

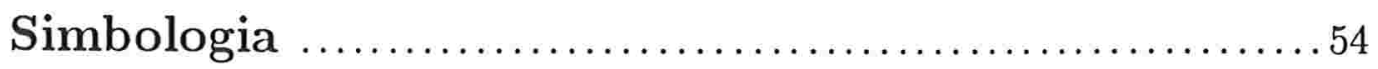

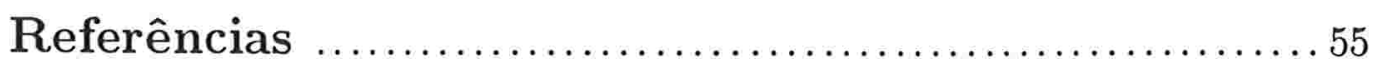




\section{Introdução}

$\mathrm{Na}$ presente tese introduzimos conceitos de diferenciabilidade Gevrey hipo-analítica para tratar da resolubilidade Gevrey local de complexos diferenciais associados a uma variedade hipo-analítica. Tais conceitos permitem, por exemplo, obter certos tipos de resolubilidade Gevrey a partir de resolubilidade clássica $\mathrm{C}^{\infty}$.

Todos os conceitos envolvidos são locais e acontecem em vizinhanças abertas de um ponto qualquer fixado na variedade, denominado origem. As análises se dão numa carta hipo-analítica $(\Omega ; Z)$ centrada na origem onde $Z=\left(Z_{1}, \ldots, Z_{m}\right)$ é uma aplicação infinitamente diferenciável, a valores em $\mathbb{C}^{m}$, com diferenciais $\mathrm{d} Z_{1}, \ldots, \mathrm{d} Z_{m} \mathbb{C}$-linearmente independentes sobre $\Omega$. Coordenadas especiais $(x, t)=\left(x_{1}, \ldots, x_{m}, t_{1}, \ldots, t_{n}\right)$ em $\Omega$ permitem expressar $Z(x, t)$ na forma $x+i \Phi(x, t)$ tornando $\Omega$ difeomorfo ao traço $\Sigma \subset \mathbb{C}^{m} \times \mathbb{R}^{n}$ da aplicação $(x, t) \stackrel{\lambda}{\mapsto}(Z(x, t), t)$. Damos especial destaque para a base $(\mathrm{M}, \mathrm{L})$ de $\mathbb{C T} \Omega$ dual da base $(\mathrm{d} Z, \mathrm{~d} t)=\left(\mathrm{d} Z_{1}, \ldots, \mathrm{d} Z_{m}, \mathrm{~d} t_{1}, \ldots, \mathrm{d} t_{n}\right)$ de $\mathbb{C T}^{*} \Omega$, constituída por derivações $\mathrm{M}_{1}, \ldots, \mathrm{M}_{m}, \mathrm{~L}_{1}, \ldots, \mathrm{L}_{n}$ satisfazendo a dualidade $\mathrm{M}_{k} t_{j}=0, \mathrm{~L}_{j} Z_{k}=0, \mathrm{M}_{k} Z_{k^{\prime}}=\delta_{k k^{\prime}}, \mathrm{L}_{j} t_{j^{\prime}}=\delta_{j j^{\prime}}$; aqui $\delta$ é a função delta de Kronecker.

O complexo diferencial associado à estrutura hipo-analítica, denotado nesta tese por $\mathbb{L}$, se realiza em $\Omega$ através dos campos $L_{1}, \ldots L_{n}$ levando $q$ formas na variável $t$ em $(q+1)$-formas na variável $t$ como abaixo:

$$
f=\sum_{|J|=q} f_{J}(x, t) \mathrm{d} t_{J} \quad \mapsto \quad \mathbb{L} f=\sum_{|J|=q-1} \sum_{j=1}^{n} \mathrm{~L}_{j} f_{J} \mathrm{~d} t_{j} \wedge \mathrm{d} t_{J}
$$

Na expressão acima, $J=\left(j_{1}<\cdots<j_{q}\right)$ e $\mathrm{d} t_{J}=\mathrm{d} t_{1} \wedge \cdots \wedge \mathrm{d} t_{j_{q}}$. Tal complexo é intrínseco à estrutura hipo-analítica e corresponde ao complexo 
de De Rham em $\mathbb{C T}^{*} \Omega$ após passagem ao quociente pelo subfibrado gerado por $\mathrm{d} Z_{1}, \ldots, \mathrm{d} Z_{m}$ (cf.[T, Sec.I.6]).

A resolubilidade local clássica de $\mathbb{L}$, caracterizada em nível $q$ pela condição dada $U \subset \Omega$ vizinhança aberta da origem existe $U^{\prime} \subset U$ vizinhança aberta da origem tal que, para toda $f \in \mathrm{C}^{\infty}\left(U ; \Lambda^{q}\right)$ com $\mathbb{L} f=0$ em $U$ existe $u \in \mathrm{C}^{\infty}\left(U^{\prime} ; \Lambda^{q-1}\right)$ com $\mathbb{L} u=f$ em $U^{\prime}$,

foi bastante estudada nestas últimas décadas, principalmente após François Treves ter conjecturado que, para $m=1$, tal condição em nível $q$ seria equivalente à condição a seguir em nível $q-1$, a saber,

dada $U \subset \Omega$ vizinhança aberta da origem existe $U^{\prime} \subset U$ vizinhança aberta da origem tal que, para todo $z_{0} \in \mathbb{C}$, a fibra $\mathcal{F}\left(U^{\prime}, z_{0}\right)$ é vazia ou o homomorfismo $\tilde{H}_{q}\left(\mathcal{F}\left(U^{\prime}, z_{0}\right)\right) \rightarrow \tilde{H}_{q}\left(\mathcal{F}\left(U, z_{0}\right)\right)$ induzido pela inclusão $\mathcal{F}\left(U^{\prime}, z_{0}\right) \hookrightarrow \mathcal{F}\left(U, z_{0}\right)$ se anula identicamente.

Aqui $\mathrm{C}^{\infty}\left(U ; \Lambda^{q}\right)$ denota $q$-formas em $\mathrm{d} t$ com coeficientes em $\mathrm{C}^{\infty}(U), \mathcal{F}\left(U, z_{0}\right)$ representa a fibra $\left\{Z(x, t)=z_{0}:(x, t) \in U\right\}$ e $\tilde{H}_{*}(\cdot)$ denota a homologia singular reduzida com coeficientes complexos. Do Teorema de BaouendiTreves segue que os germes na origem de tais fibras independem da escolha de $Z$, tornando a condição acima intrínseca à estrutura. A necessidade da condição de Treves para a resolubilidade clássica de $\mathbb{L}$ foi demonstrada por Paulo Cordaro e François Treves para todo $q$ no ano de 1991 (cf.[CT]). Já a suficiência foi demonstrada aos poucos, em casos envolvendo a regularidade da aplicação $Z$. No caso em que $Z$ é analítica, François Treves demonstrou a suficiência em $q=1$ no ano de 1983 (cf.[T1]); Paulo Cordaro e Jorge Hounie demonstraram a suficiência em $q=n$ no ano de 1991 (cf.[CH1]); Sagun Chanillo e François Treves completaram a demonstração da suficiência no ano de 1997 (cf.[ChT]). No caso geral em que $Z$ é infinitamente diferenciável, Gerardo Mendoza e François Treves demonstraram a suficiência em $q=1$ no ano de 1991 (cf.[MT]); Paulo Cordaro e Jorge Hounie demonstraram a suficiência em $q=n$ no ano de 1997 (cf.[CH2]); recentemente Paulo Cordaro e Jorge Hounie completaram a demonstração da suficiência, fechando assim a conjectura.

Nesta tese assumimos a resolubilidade local $C^{\infty}$ do complexo $\mathbb{L}$ para estabelecer sua resolubilidade em certas classes de Gevrey, denominadas hipoanalíticas. O conceito de diferenciabilidade Gevrey hipo-analítica, isto é, 
diferenciabilidade Gevrey segundo (M, L), é introduzido a partir das estimativas Gevrey na base $(M, L)$ a seguir:

$$
\sup _{\bar{U}}\left|\mathrm{M}^{\alpha} \mathrm{L}^{\gamma} f\right| \leq \mathrm{const} \cdot h^{|\alpha|+|\gamma|} \cdot(|\alpha|+|\gamma|) !^{s}, \forall(\alpha, \gamma) \in \mathbb{Z}_{+}^{m+n} .
$$

Funções $f=f(x, t)$ infinitamente diferenciáveis no fecho de vizinhanças $U \subset \subset \Omega$ da origem, cumprindo a estimativa em questão numa amplitude $h>0$, constituem espaços de Banach $\mathrm{G}_{\mathrm{ML}}^{s, h}(U)$ com a respectiva norma $\|f\|_{s, h, U}$ determinada pelo ínfimo das constantes na estimativa. Tal conceito de diferenciabilidade Gevrey de ordem $s \geq 1$ equivale ao conceito usual de ultradiferenciabilidade segundo a sequência não quase analítica $\left\{p^{!^{s}}\right\}$ para o caso Roumieu, exceto por utilizar na estimativa Gevrey a base $(\mathrm{M}, \mathrm{L})$ ao invés da base usual $\left(\mathrm{D}_{x}, \mathrm{D}_{t}\right)=\left(\partial_{x}, \partial_{t}\right)$. Como no caso usual, as inclusões $\mathrm{G}_{\mathrm{ML}}^{s, h}(U) \hookrightarrow \mathrm{G}_{\mathrm{ML}}^{s, h_{+}}(U)$ são compactas para todo $h<h_{+}$, tornando $\mathrm{G}_{\mathrm{ML}}^{s}(U)=\lim _{h \rightarrow \infty} \mathrm{G}_{\mathrm{ML}}^{s, h}(U)$ Hausdorff e Montel com a topologia de limite indutivo. O conceito de diferenciabilidade Gevrey hipo-analítica parcial adotado nesta tese é obtido combinando a diferenciabilidade Gevrey em M com a diferenciabilidade em $L$, via estimativas

$$
\forall \ell \exists C_{\ell}: \sup _{\bar{U}}\left|\mathrm{M}^{\alpha} L^{\gamma} f\right| \leq C_{\ell} \cdot h^{|\alpha|} \cdot|\alpha| !^{s}, \forall(\alpha, \gamma) \in \mathbb{Z}_{+}^{m+n},|\gamma| \leq \ell .
$$

Funções $f=f(x, t)$ infinitamente diferenciávies no fecho de vizinhanças $U \subset \subset \Omega$ da origem, cumprindo a estimativa Gevrey parcial em questão numa amplitude $h>0$, constituem espaços $\mathcal{G}_{\mathrm{ML}}^{s, h}(U)$ e a união em $h>0$ destes espaços determina o espaço $\mathcal{G}_{\mathrm{ML}}^{s}(U)$ das funções Gevrey parciais segundo $(\mathrm{M}, \mathrm{L})$.

$\mathrm{O}$ principal resultado desta tese estabelece a resolubilidade local do complexo $\mathbb{L}$ nas classes $\mathcal{G}_{\text {ML }}^{s}(U)$ a partir da resolubilidade local clássica $C^{\infty}$, para todo $s>1$ e para todo $q=1, \ldots, n$ (cf. Teorema 3.1). A técnica da demonstração (Sec.1.5 e Sec.3.3) se espelha em processos de tipo MittagLeffler e em [C] para regularizar, via transformada FBI e via operadores $P(M)$ Gevrey-diferenciais de tipo Beurling, soluções infinitamente diferenciáveis de $\mathbb{L} v=P(\mathrm{M}) f$ e obter, no final, uma solução $u$ de $\mathbb{L} u=f$ Gevrey parcial segundo $(M, L)$. Tal técnica tem importantes consequências no nível $q=1$ pois também garante a resolubilidade local de $\mathbb{L}$ nas classes $\mathrm{G}_{\mathrm{ML}}^{s}(U)$ a partir da resolubilidade local $C^{\infty}$ (cf. Sec.4.1). Com efeito segue a resolubilidade Gevrey hipo-analítica local de operadores lineares de primeira 
ordem de tipo principal satisfazendo a condição $(\mathcal{P})$ de Nirenberg-Treves (cf. Sec.4.2). É importante ressaltar que, no caso das componentes $Z_{1}, \ldots, Z_{m}$ serem Gevrey usuais de ordem $s>1$, a diferenciabilidade Gevrey segundo $(\mathrm{M}, \mathrm{L})$ de ordem $s$ coincide, módulo amplitudes, com a diferenciabilidade Gevrey usual de mesma ordem $s$. Tal coincidência, demonstrada na Proposição 1.3 , utiliza extensões $\bar{\partial}$-flat sobre o traço $\Sigma \subset \mathbb{C}^{m}$, estudadas de forma abstrata em [D] e de forma concreta nesta tese, através das classes Gevrey diferenciáveis segundo $(\mathrm{M}, \mathrm{L})$. Com efeito, os teoremas anteriormente citados se estendem naturalmente para as classes Gevrey usuais no caso particular em que $Z_{1}, \ldots, Z_{m}$ são também Gevrey usuais. Em particular segue que a condição $(\mathcal{P})$ de Nirenberg-Treves é suficiente para a resolubilidade Gervey local de operadores lineares de primeira ordem de tipo principal com coeficientes analíticos (cf. Teorema 4.2). Cabe aqui notar que, até o presente momento, é desconhecido se para um operador de ordem arbitrária $P$, de tipo principal, com coeficientes analíticos e satisfaendo a condição $(\mathcal{P})$, a equação $P u=f \operatorname{com} f$ Gevrey diferenciável de ordem $s>1$ admite solução $u$ Gevrey diferenciável de mesma ordem $s$. Resultados parciais nesta direção podem ser consultados em [R] e [GP].

O último resultado que destacamos nesta introdução diz respeito ao teorema de aproximação de Baouendi-Treves, que garante a aproximação holomorfa de soluções infinitamente diferenciáveis com respeito a $L_{1}, \ldots, L_{n}$ na topologia de $C^{\infty}$. Nosso teorema (cf. Sec.2.4) estabelece a aproximação holomorfa de soluções Gevrey hipo-analíticas de ordem $s>1$ na topologia de $\mathrm{G}_{\mathrm{ML}}^{s}(U)$ utilizando fórmulas de aproximação por gaussianas. Observamos que tal aproximação já havia sido demonstrada em [D] no caso Gevrey usual sem o uso de gaussianas; no nosso entendimento o uso de gaussianas simplifica por demais a demonstração. 


\section{Capítulo 1}

\section{Hipo-analiticidade Gevrey em codimensão nula}

Este capítulo trata de questões envolvendo a diferenciabilidade Gevrey de ordem $s>1$ segundo uma estrutura hipo-analítica de codimensão nula numa variedade diferenciável $m$-dimensional.

\subsection{Estruturas hipo-analíticas com codimen- são nula: a base $\mathrm{M}$}

Seja $\mathcal{M}$ uma variedade diferenciável $m$-dimensional equipada com uma estrutura hipo-analítica de codimensão nula (cf. [T, Def.III.1.1]). Tal estrutura é caracterizada pela existência de uma coleção de cartas hipo-analíticas $\left(B_{\imath}, Z_{\imath}\right)$ onde $B_{\imath}$ são abertos cobrindo $\mathcal{M}$ e $Z_{\imath}=\left(Z_{\imath 1}, \ldots, Z_{\imath m}\right)$ são aplicações infinitamente diferenciáveis em $B_{\imath}$, a valores em $\mathbb{C}^{m}$, satisfazendo

- para cada $\imath$, as diferenciais $\mathrm{d} Z_{\imath 1}, \ldots, \mathrm{d} Z_{\imath m}$ são $\mathbb{C}$-linearmente independentes em cada ponto de $B_{i}$;

- para cada par $\imath \neq \kappa$ e para cada $P \in B_{\imath} \cap B_{\kappa}$, existe um biholomorfismo $H$ de uma vizinhança aberta de $Z_{\imath}(P) \subset \mathbb{C}^{m}$ numa vizinhança aberta de $Z_{\kappa}(P) \subset \mathbb{C}^{m}$ tal que $Z_{\kappa}=H \circ Z_{\imath}$ numa vizinhança de $P$.

Observamos que as condições acima tornam cada $Z_{\imath}\left(B_{\imath}\right)$ imersas numa subvariedade real maximal de $\mathbb{C}^{m}$. 
Fixado um ponto 0 qualquer na variedade, doravante denominado origem, vamos utilizar [T, II.1. The Coarse Local Embedding] e biholomorfismos que preservam a origem para trabalhar numa situável carta hipo-analítica $(B, Z)$ centrada na origem, tornando o aberto $B$ difeomorfo a uma subvariedade real maximal $\Sigma \subset \mathbb{C}^{m}$ bem posicionada na origem de $\mathbb{C}^{m}$. Mais precisamente, vamos trabalhar em coordenadas especiais

$$
x=\left(x_{1}, \ldots, x_{m}\right), x(0)=0, B=\{x:\|x\|<d\}
$$

nas quais

$$
Z_{k}(x)=x+i \Phi_{k}(x), k=1, \ldots, m
$$

com $\Phi=\left(\Phi_{1}, \ldots, \Phi_{m}\right)$ infinitamente diferenciável em $B$, a valores em $\mathbb{R}^{m}$, satisfazendo

$$
\Phi(0)=0, \Phi_{x}(0)=0, \quad\left\|\Phi_{x}\right\|<\frac{1}{2} \text { em } B .
$$

Aqui $\Phi_{x}(x)$ representa a matriz quadrada $m \times m$ com entradas na linha $k$ e coluna $k^{\prime}$ dadas por $\frac{\partial \Phi_{k}}{\partial x_{k^{\prime}}}(x)$. Observe que a matriz $Z_{x}=\mathrm{Id}+\Phi_{x}$ é não singular em todos os pontos de $B$, o que permite introduzir a base

$$
\mathrm{M}=\left(\mathrm{M}_{1}, \ldots, \mathrm{M}_{m}\right)
$$

do fibrado tangente complexo $\mathbb{C T} \mathcal{M}$ em $B$ composta pelas derivações

$$
\mathrm{M}_{k}=\sum_{k^{\prime}=1}^{m} \mu_{k k^{\prime}}(x) \frac{\partial}{\partial x_{k^{\prime}}}, k=1, \ldots, m
$$

onde $\left(\mu_{k k^{\prime}}\right)$ é a inversa da transposta de $Z_{x}$. Importante ressaltar que

$$
\mathrm{M}_{k} Z_{k}=1, \mathrm{M}_{k} Z_{k^{\prime}}=0 \text { se } k \neq k^{\prime}
$$

e que

$$
\Sigma=\{Z(x)=x+i \Phi(x): x \in B\}
$$

\subsection{Diferenciabilidade Gevrey segundo M}

$\mathrm{O}$ conceito de diferenciabilidade Gevrey segundo $\mathrm{M}$ de ordem $s \geq 1$, no fecho de uma vizinhança $V \subset B$ da origem e numa amplitude $h>0$, advém da estimativa

$$
\sup _{\bar{V}}\left|\mathrm{M}^{\alpha} f\right| \leq \mathrm{const} \cdot h^{|\alpha|}|\alpha| !^{s}, \forall \alpha \in \mathbb{Z}_{+}^{m}
$$


onde $|\alpha|=\alpha_{1}+\cdots+\alpha_{m}$ e $\mathbf{M}^{\alpha}=\mathrm{M}_{1}^{\alpha_{1}} \cdots \mathrm{M}_{m}^{\alpha_{m}}$. Tal conceito equivale ao conceito usual de ultradiferenciabilidade com respeito à sequência não quase analítica $\left\{p !^{\mid s}\right\}$ para o caso Roumieu (cf. [K]) exceto por utilizar em (1.8) a base $M=\left(M_{1}, \ldots, M_{m}\right)$ ao invés da base usual $D=\left(\frac{\partial}{\partial x_{1}}, \ldots, \frac{\partial}{\partial x_{m}}\right)$.

Definição 1.1 Dizemos que uma função $f=f(x)$ infinitamente diferenciável no fecho de uma vizinhança $V \subset \subset B$ da origem é de classe $s$-Gevrey segundo $\mathrm{M}$ em $V$ numa amplitude $h>0$ quando $f$ cumpre a estimativa (1.8) para alguma constante positiva. O subespaço de $\mathrm{C}^{\infty}(\bar{V}){ }^{1}$ constituído por tais funções será denotado por $\mathrm{G}_{\mathrm{M}}^{s, h}(V)$.

Como em [K, (2.4)], cada $\mathrm{G}_{\mathrm{M}}^{s, h}(V)$ é um espaço de Banach em relação à norma definida pelo ínfimo das constantes em (1.8), a saber,

$$
\|f\|_{s, h, V}=\sup \left\{\frac{\left|\mathrm{M}^{\alpha} f(x)\right|}{h^{|\alpha|}|\alpha| !^{s}}: x \in \bar{V}, \alpha \in \mathbb{Z}_{+}^{m}\right\} .
$$

Explicitando,

$$
\mathrm{G}_{\mathrm{M}}^{s, h}(V)=\left\{f \in \mathrm{C}^{\infty}(\bar{V}):\|f\|_{s, h, V}<\infty\right\} .
$$

Proposição 1.1 Para todo $V \subset \subset B$ e para todo $s \geq 1$ as inclusões

$$
\mathrm{G}_{\mathrm{M}}^{s, h}(V) \hookrightarrow \mathrm{G}_{\mathrm{M}}^{s, h_{+}}(V), h<h_{+}
$$

são compactas

Demonstração :

$\triangleright \quad$ Basta mostrarmos que a bola unitária $\mathcal{B}=\left\{\|f\|_{s, h, V} \leq 1\right\} \subset \mathrm{G}_{\mathrm{M}}^{s, h}(V)$ é totalmente limitada em $\mathrm{G}_{\mathrm{M}}^{s, h_{+}}(V)$.

Fixemos $\varepsilon>0$.

Como $\mathcal{B}$ é totalmente limitada em $C^{\ell}(\bar{V})$ pelo Teorema de Ascoli, para cada $\ell$ fixado existem finitos $f_{1}, \ldots, f_{\nu} \in \mathcal{B}$ tais que, para toda $f \in \mathcal{B}$ existe algum $\nu_{0} \in\{1, \ldots, \nu\}$ com

$$
\sup _{\bar{V}}\left|M^{\alpha}\left[f-f_{\nu_{0}}\right]\right| \leq \varepsilon h_{+}^{|\alpha|}|\alpha| !^{s}, \forall|\alpha| \leq \ell .
$$

\footnotetext{
${ }^{1}$ Nesta tese $C^{\infty}(K)$ denota o espaço das funções infinitamente diferenciáveis no interior de $K$ cujas derivadas se estendem continuamente para a fronteira de $K$.
} 
Com relação às derivações de ordem $|\alpha|>\ell$, tomando $\ell$ grande tal que $\left(h / h_{+}\right)^{\ell}<\varepsilon / 2$ segue, via desigualdade triangular, que

$$
\begin{aligned}
\sup _{\bar{V}}\left|M^{\alpha}\left[f-f_{\nu_{0}}\right]\right| & \leq 2 h^{|\alpha|}|\alpha| !^{s} \leq \varepsilon h^{|\alpha|-\ell} h_{+}^{\ell}|\alpha| !^{s}, \forall \alpha \\
& \leq \varepsilon h_{+}^{|\alpha|-\ell} h_{+}^{\ell}|\alpha| !^{s}=\varepsilon h_{+}^{|\alpha|}|\alpha| !^{s}, \forall|\alpha|>\ell .
\end{aligned}
$$

Definição 1.2 Dizemos que uma função $f$ é de classe s-Gevrey segundo $\mathrm{M}$ numa vizinhança $V \subset \subset B$ da origem quando $f$ pertence $a \mathrm{G}_{\mathrm{M}}^{s, h}(V)$ para alguma amplitude $h>0$. O subespaço de $\mathrm{C}^{\infty}(\bar{V})$ constituído por tais funções será denotado por $\mathrm{G}_{\mathrm{M}}^{s}(V)$.

Segue da Proposição 1.1 e de [Bo] que os espaços

$$
\mathrm{G}_{\mathrm{M}}^{s}(V)=\underset{h \lim _{\infty}}{\longrightarrow} \mathrm{G}_{\mathrm{M}}^{s, h}(V)
$$

são Hausdorff e Montel com a topologia de limite indutivo. Em particular, sequências limitadas em algum $\mathrm{G}_{\mathrm{M}}^{s, h}(V)$ admitem subsequências convergentes em $\mathrm{G}_{\mathrm{M}}^{s, h_{+}}(V)$ para todo $h_{+}>h$ e, consequentemente, em $\mathrm{G}_{\mathrm{M}}^{s}(V)$.

Vamos denotar por $G_{M}^{s}(0)$ o espaço dos germes na origem de funções $s$ Gevrey segundo $M$, caracterizados pela relação de equivalência que identifica duas funções $s$-Gevrey segundo $\mathrm{M}$ coincidentes no fecho de alguma vizinhança da origem. Só para evidenciar,

$$
\mathrm{G}_{\mathrm{M}}^{s}(0)=\left\{\text { classes } \mathbf{f} \text { representadas por } f \in \mathrm{G}_{\mathrm{M}}^{s}(V), 0 \in V \subset \subset B\right\}
$$

Observação: as classes de funções Gevrey usuais, denotadas nesta tese por $\mathrm{G}^{s, h}(V), \mathrm{G}^{s}(V)$ e $\mathrm{G}^{s}(0)$, são aquelas onde $\mathrm{M}=\mathrm{D}$ ou, equivalentemente, $Z(x)=x$.

Como veremos a seguir, as funções em $\mathrm{G}_{\mathrm{M}}^{s}(V)$ de ordem $s>1$ podem ser caracterizadas em $\mathbb{C}^{m}$ através de funções infinitamente diferenciáveis em vizinhanças complexas de $Z(V)$ que são $\bar{\partial}$-flat sobre $\Sigma$. Para tal vamos considerar funções $F=F(z)$ infinitamente diferenciáveis no fecho de vizinhanças $\mathcal{U} \subset \subset \mathbb{C}^{m}$ de $Z(V)$ com $\bar{\partial} F \sim_{\Sigma} 0^{2}$, isto é, com $\frac{\partial F}{\partial \bar{z}_{k}}$ se anulando de

\footnotetext{
${ }^{2}$ Vamos usar a notação $\sim_{\Sigma} 0$ para caracterizar anulamento de ordem $\infty$ sobre $\Sigma$.
} 
ordem infinita em $\Sigma \cap \mathcal{U}$ para todo $k=1, \ldots, m$. Dentre elas destacamos as Gevrey usuais em $\mathcal{U}$, nas classes $\mathrm{G}^{s}(\mathcal{U})$, definidas a partir da estimativa (1.8) na base $\left(\partial_{z}, \bar{\partial}_{z}\right)=\left(\frac{\partial}{\partial z_{1}}, \cdots, \frac{\partial}{\partial z_{m}}, \frac{\partial}{\partial \bar{z}_{1}}, \cdots, \frac{\partial}{\partial \bar{z}_{m}}\right)$, a saber,

$$
\sup _{\bar{u}}\left|\partial_{z}^{\alpha} \partial_{\bar{z}}^{\beta} F\right| \leq \text { const } \cdot h^{|\alpha|+|\beta|}(|\alpha|+|\beta|) !^{s}, \forall \alpha, \beta \in \mathbb{Z}_{+}^{m} .
$$

Tal estimativa se traduz sobre $\Sigma$, via $\bar{\partial} F \sim_{\Sigma} 0$, em

$$
\sup _{\Sigma \bar{n} \bar{u}}\left|\partial_{z}^{\alpha} F\right| \leq \text { const } \cdot h^{|\alpha|}|\alpha| !^{s}, \forall \alpha \in \mathbb{Z}_{+}^{m} .
$$

Definição 1.3 Vamos dentotar por $\mathrm{G}_{\mathrm{M}}^{s}(\mathcal{U})$ o espaço das funções $F=F(z)$ infinitamente diferenciáveis no fecho de uma vizinhança $\mathcal{U} \subset \subset \mathbb{C}^{m}$ da origem com $\bar{\partial} F \sim_{\Sigma} 0$ e satisfazendo a estimativa (1.14). Só para evidenciar,

$$
\mathrm{G}_{\mathrm{M} \star}^{s}(\mathcal{U})=\left\{F \in \mathrm{C}^{\infty}(\overline{\mathcal{U}}) \text { com } \bar{\partial} F \sim_{\Sigma} 0 \text { e satisfazendo }(1.14)\right\} .
$$

A caracterização em $\mathbb{C}^{m}$ das funções Gevrey diferenciáveis segundo $M$ de ordem $s>1$ se dá através do seguinte resultado:

Teorema 1.1 Se $f$ é infinitamente diferenciável no fecho de uma vizinhança $V \subset \subset B$ da origem e se $s>1$ então são equivalentes:

(i) $f \in \mathrm{G}_{\mathrm{M}}^{s}(V)$

(ii) existem $\mathcal{U} \supset Z(V)$ e $\left.F \in \mathrm{G}_{\mathrm{M}_{*}}^{s}(\mathcal{U}) \operatorname{com} f\right|_{V}=\left.F \circ Z\right|_{V}$.

(iii) existem $\mathcal{U} \supset Z(V)$ e $F \in G^{s}(\mathcal{U})$ com $\left.f\right|_{V}=\left.F \circ Z\right|_{V}$ e com $\bar{\partial} F \sim_{\Sigma} 0$.

(iv) existe $F \in \mathrm{G}^{s}\left(\mathbb{C}^{m}\right)$ com $\left.f\right|_{V}=\left.F \circ Z\right|_{V}$ e com $\bar{\partial} F \sim_{\Sigma} 0$. 


\section{Demonstração :}

$\triangleright$ As implicações $(i v) \Rightarrow(i i i) \Rightarrow(i i)$ são imediatas.

A implicação $(i i) \Rightarrow$ (i) é uma consequência da aplicação da regra da cadeia junto com a dualidade (1.6) e com o anulamento $\bar{\partial} F \sim_{\Sigma} 0$, pois nestas condições vale a igualdade

$$
\left|\mathrm{M}^{\alpha}[F \circ Z]\right|=\left|\partial_{z}^{\alpha}[F] \circ Z\right|
$$

e (1.14) implica (1.8).

A implicação $(i) \Rightarrow($ ii $)$ segue encontrando uma solução $F=F(z)$ infinitamente diferenciável para o problema

$$
\mid \begin{aligned}
& \bar{\partial} F \sim_{\Sigma} 0 \\
& \left.F\right|_{\Sigma}=f
\end{aligned}
$$

Por fim, as implicações $(i i) \Rightarrow(i i i) \Rightarrow(i v)$ decorrem da seguinte variante fraca do Teorema de extensão de Whitney para classes de funções não quase-analíticas, demonstrado por Joaquim Bruna em [B, Teo.3.1]:

qualquer jato de Whitney Gevrey de ordem $s>1$ sobre um compacto $\mathcal{K}$ de $\mathbb{R}^{2 m}$ se estende a uma função em $\mathrm{G}^{s}\left(\mathbb{R}^{2 m}\right)$.

Em [B], jatos de Whitney Gevrey sobre compactos $\mathcal{K} \subset \subset \mathbb{R}^{2 m}$ são sequências de funções $\mathrm{G}=\left(G^{\gamma}\right)$, indexadas em $\gamma \in \mathbb{Z}_{+}^{2 m}$, cumprindo as estimativas a seguir para todo $y, y^{\prime} \in \mathcal{K}, \gamma \in \mathbb{Z}_{+}^{2 m} \mathrm{e} \ell \in \mathbb{Z}_{+}$, a saber,

$$
\begin{aligned}
\left|G^{\gamma}(y)\right| & \leq C_{1} h_{1}^{|\gamma|}|\gamma| !^{s} \\
\left|\left(\mathbf{R}_{y}^{\ell} G\right)_{\gamma}\left(y^{\prime}\right)\right| & \leq C_{2} \frac{\left|y-y^{\prime}\right|^{\ell-|\gamma|+1}}{(\ell-|\gamma|+1) !} h_{2}^{\ell+1}(\ell+1) !^{s},|\gamma| \leq \ell ;
\end{aligned}
$$

aqui $C_{1}, C_{2}, h_{1}, h_{2}$ são constantes positivas e $\left(\mathbf{R}_{y}^{\ell} G\right)_{\gamma}\left(y^{\prime}\right)$ se expressa por

$$
\left(\mathbf{R}_{y}^{\ell} G\right)_{\gamma}\left(y^{\prime}\right)=G^{\gamma}\left(y^{\prime}\right)-\sum_{\left|\gamma+\gamma^{\prime}\right| \leq \ell} \frac{G^{\gamma+\gamma^{\prime}}(y)}{\gamma^{\prime} !}\left(y^{\prime}-y\right)^{\gamma^{\prime}} .
$$

Identificando $\mathbb{R}^{2 m}$ com $\mathbb{C}^{m}$ e $\gamma \operatorname{com}(\alpha, \beta) \in \mathbb{Z}^{m+m}$, a condição (ii) determina o seguinte jato de Whitney Gevrey sobre $\Sigma$, a saber,

$$
F^{\gamma}(Z)=\partial_{z}^{\alpha} \partial_{\bar{z}}^{\beta} F(Z)=\mid \begin{array}{ccc}
\partial_{z}^{\alpha} F(Z) & \text { se } \beta=0 \\
0 & \text { se } \beta \neq 0
\end{array} .
$$


Observe que (1.14) garante a primeira caracterização (1.15) de Jato de Whitney Gevrey para $F=\left(F^{\gamma}\right)$. Com relação à segunda caracterização, basta observar que

$$
\left(\mathbf{R}_{Z}^{\ell} F\right)_{\gamma}\left(Z^{\prime}\right)=\mid \begin{array}{r}
\partial_{z}^{\alpha} F\left(Z^{\prime}\right)-\sum_{\left|\alpha^{\prime}\right| \leq \ell-|\alpha|} \frac{\partial_{z}^{\alpha+\alpha^{\prime}} F(Z)}{\alpha^{\prime} !}\left(Z^{\prime}-Z\right)^{\alpha^{\prime}} \\
\text { se } \beta=0 \\
0 \text { se } \beta \neq 0
\end{array}
$$

é precisamente o resto de ordem $\ell-|\alpha|+1$ da expansão em série de Taylor de $\partial_{z}^{\alpha} F\left(Z^{\prime}\right)$ no ponto $Z$; as estimativas para tal resto garantem que

$$
\left|\left(\mathbf{R}_{Z}^{\ell} F\right)_{\gamma}\left(Z^{\prime}\right)\right| \leq \frac{\sup _{\Sigma}\left|\partial_{z}^{\ell-\alpha+1} F\right|}{(\ell-|\alpha|+1) !}\left|Z^{\prime}-Z\right|^{\ell-|\alpha|+1}
$$

e (1.14) garante (1.16).

Passemos agora a uma outra caracterização das classes de funções Gevrey diferenciáveis segundo $M$, que utiliza os espaços das funções infinitamente diferenciáveis na origem de $\mathbb{C}^{m}$ que são flat sobre $\Sigma$.

Definição 1.4 Vamos denotar por $\mathcal{F}_{\Sigma}(\mathcal{U})$ o espaço das funções $F=F(z)$ infinitamente diferenciáveis no fecho de uma vizinhança $\mathcal{U} \subset \subset \mathbb{C}^{m}$ da origem que se anulam de ordem infinita sobre $\Sigma$, isto é,

$$
\mathcal{F}_{\Sigma}^{\bullet}(\mathcal{U})=\left\{F \in C^{\infty}(\overline{\mathcal{U}}) \operatorname{com} F \sim_{\Sigma} 0\right\}
$$

O espaço dos germes na origem de tais funções será denotado por $\mathcal{F}_{\Sigma}^{\bullet}(0)$

Proposição 1.2 Para todo $s>1$ a sequência

$$
0 \rightarrow \mathcal{F}_{\Sigma}^{\bullet}(0) \longrightarrow \mathrm{G}_{\mathrm{M} *}^{s}(0) \longrightarrow \mathrm{G}_{\mathrm{M}}^{s}(0) \rightarrow 0
$$

é uma sequência exata curta, garantindo assim o isomorfismo

$$
\mathrm{G}_{\mathrm{M}}^{s}(0) \simeq \mathrm{G}_{\mathrm{M}_{*}(0)}^{s} / \mathcal{F}_{\Sigma}(0)
$$




\section{Demonstração :}

$\triangleright \quad$ Basta invocarmos o Teorema 1.1 e mostrarmos que o núcleo da aplicação $\mathrm{G}_{\mathrm{M}_{*}}^{s}(0) \rightarrow \mathrm{G}_{\mathrm{M}}^{s}(0)$ é precisamente $\mathcal{F}_{\Sigma}^{\circ}(0)$, já que sua sobrejetividade decorre diretamente da proposição invocada.

Observe que o núcleo em questão contém $\mathcal{F}_{\Sigma}^{\bullet}(0)$, já que o item (ii) da Proposição 1.1 garante que o mesmo é constituído pelas soluções $F=F(z)$ infinitamente diferenciáveis do problema formal de Cauchy

$$
\mid \begin{aligned}
& \bar{\partial} F \sim_{\Sigma} 0 \\
& \left.F\right|_{\Sigma}=0
\end{aligned}
$$

A unicidade das soluções para tal problema garante a inclusão recíproca, isto é, garante que $F \sim_{\Sigma} 0$.

Cabe observar aqui que, fixada duas cartas hipo-analíticas $(B, Z)$ e $\left(B^{\prime}, Z^{\prime}\right)$, a composição com o biholomorfismo $Z^{\prime}=H(Z)$ existente induz um isomorfismo entre os espaços correspondentes $G_{M *}^{s}(0)$ e $G_{M_{*^{\prime}}}^{s}(0)$ e também entre os espaços correspondentes $\mathcal{F}_{\Sigma}^{\bullet}(0)$ e $\mathcal{F}_{\Sigma^{\prime}}^{\bullet}(0)$, tornando-os assim invariantes hipoanalíticos. Com efeito, o quociente em (1.18) também será um invariante hipo-analítico.

Finalizamos esta seção estabelecendo a equivalência entre os conceitos de diferenciabilidade Gevrey usual e diferenciabilidade Gevrey segundo M no caso particular em que $\Sigma$ é uma subvariedade Gevrey, isto é, no caso particular em que as componentes $Z_{1}, \ldots, Z_{m}$ da carta hipo-analítica $(B, Z)$ são funções Gevrey usuais em $B$.

Proposição 1.3 Se $\Sigma$ é uma subvariedade Gevrey de ordem $s>1$ então $\mathrm{G}_{\mathrm{M}}^{s}(0)=\mathrm{G}^{s}(0)$.

\section{Demonstração :}

D A caracterização (iv) do Teorema 1.1, mais o fato da diferenciabilidade Gevrey ser invariante por composição, garante a inclusão de $G_{M}^{s}(0)$ em $G^{s}(0)$, já que os germes $f \in G_{M}^{s}(0)$ podem ser representados pela função $F \circ Z(x) \operatorname{com} F$ e $Z$ Gevrey usuais. 
A inclusão recíproca é garantida por [D, Prop.2.1], que estabelece a seguinte extensão local $\bar{\partial}$-flat sobre $\Sigma$ :

se $\Sigma$ é uma subvariedade real maximal Gevrey e se $f \in G^{s}(V)$ então para todo $x_{0} \in V$ existe uma vizinhança $\mathcal{U}$ de $Z\left(x_{0}\right)$ em $\mathbb{C}^{m}$ e $\left.F \in \mathrm{G}^{s}(\mathcal{U}) \operatorname{com} F \circ Z\right|_{U}=f$ e $\operatorname{com} \bar{\partial} F \sim_{\Sigma \cap Z(V)}=0$.

Fixada $\mathbf{f} \in \mathrm{G}^{s}(0)$, tal extensão local garante a existência de $\mathbf{F} \in \mathrm{G}_{\mathrm{M} *}^{s}(0)$ $\left.\operatorname{com} F\right|_{\Sigma}=f$ e a Proposição 1.1 garante que $\mathbf{f} \in \mathrm{G}_{\mathrm{M}}^{s}(0)$.

\subsection{Exemplos de funções Gevrey segundo $M$}

Em princípio a dualidade (1.6) determina que as componentes $Z_{1}, \ldots, Z_{m}$ da carta hipo-analítica $(B, Z)$ são funções em $\mathrm{G}_{\mathrm{M}}^{1, h}(V)$ para toda vizinhança aberta $V \subset \subset B$ da origem e para toda amplitude $h>0$, inclusive no caso em que tais componentes são apenas infinitamente diferenciávies.

Outros exemplos neste sentido podem ser obtidos através da composição da função $Z$ com qualquer função inteira em $\mathbb{C}^{m}$, como no caso da função colchete quadrado, a saber,

$$
\langle Z(x)\rangle^{2}=\left(Z_{1}(x)\right)^{2}+\cdots+\left(Z_{m}(x)\right)^{2} .
$$

Para os demais exemplos vamos fixar um valor $p>0$ e considerar funções $f_{p} \in C^{\infty}(B)$ se anulando de ordem infinita na origem, definidas como segue:

$$
f_{p}(x)=\left\{\begin{array}{ccc}
e^{-1 /\left((z(x))^{2}\right)^{p}} & \text { se } x \neq 0 \\
0 & \text { se } x=0
\end{array} .\right.
$$

A determinação da regularidade Gevrey segundo $M$ de $f_{p}$ passa pela estimativa (1.8) no fecho de alguma vizinhança $V \subset \subset B$ da origem, posto que $f_{p} \in \mathrm{G}_{\mathrm{M}}^{1}(B \backslash V)$. Neste sentido vamos considerar vizinhanças $V \subset \subset B$ suficientemente próximas da origem para validar, via (1.3), estimativas do tipo

$$
\begin{aligned}
\operatorname{Re}\langle Z(x)\rangle^{2} & =\|x\|^{2}-\|\Phi(x)\|^{2}>(1-\epsilon)\|x\|^{2}, \\
\left|\operatorname{Im}\langle Z(x)\rangle^{2}\right| & =2|x \cdot \Phi(x)|<2 \epsilon\|x\|^{2}
\end{aligned}
$$


num pequeno valor de $\epsilon$ a ser escolhido posteriormente. Vamos agora considerar discos complexos

$$
\Gamma_{\rho}(z)=\left\{\zeta \in \mathbb{C}:\left|\zeta-\langle z\rangle^{2}\right| \leq \rho \operatorname{Re}\langle z\rangle^{2}\right\}
$$

e escolher $V$ e $\rho$ pequenos para validar

$$
\operatorname{Re} \zeta^{-p}>\frac{1}{2\left(\operatorname{Re}\langle z\rangle^{2}\right)^{p}}, \forall \zeta \in \Gamma_{\rho}(z), \forall z \in Z(\bar{V} \backslash\{0\}) .
$$

A existência de $V$ e $\rho$ advém do fato dos elementos em $\Gamma_{\rho}(z)$ se expressarem na forma

$$
\zeta=\operatorname{Re}\langle z\rangle^{2}\left(1+\rho e^{i \theta}+i \eta\right) \quad, \quad|\eta|=\left|\frac{\operatorname{Im}\langle z\rangle^{2}}{\operatorname{Re}\langle z\rangle^{2}}\right| \leq \frac{2 \epsilon}{1-\epsilon}
$$

validando a estimativa

$$
\operatorname{Re} \zeta^{-p}=\frac{\operatorname{Re} \bar{\zeta}^{p}}{|\zeta|^{2 p}}=\frac{1}{\left(\operatorname{Re}\langle z\rangle^{2}\right)^{p}} \frac{\operatorname{Re}\left(1+\rho e^{-i \theta}-i \eta\right)^{p}}{\left|1+\rho e^{i \theta}+i \eta\right|^{2 p}} \geq \frac{1}{\left(\operatorname{Re}\langle z\rangle^{2}\right)^{p}} \cdot \psi(\rho, \eta)
$$

onde

$$
\psi(\rho, \eta)=\frac{\max _{\theta} \operatorname{Re}\left(1+\rho e^{-i \theta}-i \eta\right)^{p}}{\min _{\theta}\left|1+\rho e^{i \theta}+i \eta\right|^{2 p}}
$$

a continuidade de $\psi$ na origem determina a existência de $\delta>0$ tal que $|\rho|<\delta$ e $|\eta|<\delta$ implicam $\psi(\rho, \eta)>1 / 2$; basta escolher $\epsilon<\frac{\delta}{2+\delta}$ e considerar $\rho=\delta / 2$. Passemos agora a estimar $\left|\mathrm{M}^{\alpha} f_{p}\right|$ no fecho de $V$. Como $e^{-\zeta^{-p}}$ é holomorfa na imagem de $\langle z\rangle^{2}$ para todo $z$ em $Z(\bar{V} \backslash\{0\})$,

$$
\mathbf{M}_{k} f_{p}=\frac{\partial}{\partial \zeta}\left[e^{-\zeta^{-p}}\right]\left(\langle z\rangle^{2}\right) \mathbf{M}_{k}\left[\langle z\rangle^{2}\right]=2 z_{k} \frac{\partial}{\partial \zeta}\left[e^{-\zeta^{-p}}\right]\left(\langle z\rangle^{2}\right) .
$$

Assumindo $|z| \leq 1$ em $Z(\bar{V})$ segue que

$$
\sup _{\bar{V}}\left|\mathrm{M}^{\alpha} f_{p}\right| \leq \sup _{z \in Z(\bar{V} \backslash\{0\})} \sum_{\alpha^{\prime} \leq \alpha} 2^{\left|\alpha-\alpha^{\prime}\right|}\left|\partial_{\zeta}^{\left|\alpha^{\prime}\right|}\left[e^{-\zeta^{-p}}\right]\left(\langle z\rangle^{2}\right)\right|
$$


Através das estimativas de Cauchy,

$$
\begin{aligned}
\sup _{z \in Z(\bar{V} \backslash\{0\})}\left|\partial_{\zeta}^{\ell}\left[e^{-\zeta^{-p}}\right]\left(\langle z\rangle^{2}\right)\right| & =\sup _{z \in Z(\bar{V} \backslash\{0\})}\left|\frac{\ell !}{2 \pi i} \int_{\Gamma_{\rho}(z)} \frac{e^{-\zeta^{-p}}}{\left(\rho \operatorname{Re}\langle z\rangle^{2}\right)^{\ell+1}} d \zeta\right| \\
& \leq \sup _{\substack{\zeta \in \Gamma_{\rho}(z) \\
z \in Z(\bar{V} \backslash\{0\})}} \frac{\ell !}{\left(\rho \operatorname{Re}\langle z\rangle^{2}\right)^{\ell}} e^{-\operatorname{Re} \zeta^{-p}} \\
& \leq \ell ! \rho^{-\ell} \sup _{z \in Z(\bar{V} \backslash\{0\})} \frac{e^{-1 / 2\left(\operatorname{Re}\langle z\rangle^{2}\right)^{p}}}{\left(\operatorname{Re}\langle z\rangle^{2}\right)^{\ell}}
\end{aligned}
$$

e como a função $\frac{e^{-1 / 2 \xi^{p}}}{\xi^{\ell}}$ assume seu valor máximo em $\xi=\left(\frac{p}{2 \ell}\right)^{1 / p}$ temos

$$
\begin{aligned}
\sup _{z \in Z(\bar{V} \backslash\{0\})}\left|\partial_{\zeta}^{\ell}\left[e^{-\zeta^{-p}}\right]\left(\langle z\rangle^{2}\right)\right| & \leq \ell ! \rho^{-\ell}\left(\frac{p}{2 \ell}\right)^{-\ell / p} e^{-\ell / p} \\
& =\ell ! \rho^{-\ell}\left(\frac{p e}{2}\right)^{-\ell / p}\left(\ell^{\ell}\right)^{1 / p} \\
& \leq \ell ! \rho^{-\ell}\left(\frac{p e}{2}\right)^{-\ell / p}\left(\ell ! e^{\ell}\right)^{1 / p} \\
& =\left[\left(\frac{2}{p \rho^{p}}\right)^{1 / p}\right]^{\ell} \cdot \ell !^{1+1 / p}
\end{aligned}
$$

Concluímos então que

$$
\begin{aligned}
\sup _{x \in \bar{V}}\left|\mathrm{M}^{\alpha} f_{p}(x)\right| & \leq \sum_{\alpha^{\prime} \leq \alpha} 2^{\left|\alpha-\alpha^{\prime}\right|}\left[\left(2 /\left(p \rho^{p}\right)\right)^{1 / p}\right]^{\left|\alpha^{\prime}\right|} \cdot\left|\alpha^{\prime}\right| !^{1+1 / p} \\
& \leq\left(\sum_{\alpha^{\prime} \leq \alpha} 2^{\left|\alpha-\alpha^{\prime}\right|}\left[\left(2 /\left(p \rho^{p}\right)\right)^{1 / p}\right]^{\left|\alpha^{\prime}\right|}\right) \cdot|\alpha| !^{1+1 / p} \\
& =\left[2+\left(2 /\left(p \rho^{p}\right)\right)^{1 / p}\right]^{|\alpha|} \cdot|\alpha| !^{1+1 / p}, \forall \alpha \in \mathbb{Z}_{+}^{m}
\end{aligned}
$$

Fica demonstrada a seguinte proposição.

Proposição 1.4 Para todo $p>0$ o germe $\mathrm{f}_{p}$ de $(1.20)$ pertence a $\mathrm{G}_{\mathrm{M}}^{1+1 / p}(0)$. 


\subsection{Fórmulas de aproximação}

Nesta seção apresentamos fórmulas de aproximação por gaussianas para classes de funções Gevrey diferenciáveis segundo $M$ de ordem $s>1$.

Como em [T, (II.2.4)] vamos considerar funções $\tilde{\mathcal{E}}_{\tau}(\chi f)$ inteiras em $\mathbb{C}^{m}$, indexadas num parâmetro $\tau>0$, definidas a partir de uma função $f \in$ $\mathrm{G}_{\mathrm{ML}}^{s, h}(V)(s>1)$ e de uma função de corte $\chi \in \mathrm{C}_{c}^{\infty}(V)$ com $\chi \equiv 1 \mathrm{em}$ $V^{\prime} \subset \subset V$; mais precisamente,

$$
\tilde{\mathcal{E}}_{\tau}(\chi f)(z)=\left(\frac{\tau}{\pi}\right)^{m / 2} \int e^{-\tau\left(z-Z\left(x^{\prime}\right)\right\rangle^{2}} \chi\left(x^{\prime}\right) f\left(x^{\prime}\right) \mathrm{d} Z\left(x^{\prime}\right) .
$$

Na fórmula, $\mathrm{d} Z\left(x^{\prime}\right)=\mathrm{d} Z_{1}\left(x^{\prime}\right) \wedge \cdots \wedge \mathrm{d} Z_{m}\left(x^{\prime}\right)=\operatorname{det} Z_{x}\left(x^{\prime}\right) \mathrm{d} x^{\prime}{ }_{1} \wedge \cdots \wedge \mathrm{d} x^{\prime}{ }_{m}$. Vamos considerar também as respectivas restrições a $\Sigma$, a saber,

$$
\mathcal{E}_{\tau}(\chi f)(x)=\tilde{\mathcal{E}}_{\tau}(\chi f)(Z(x)) .
$$

Teorema 1.2 Para toda $V^{\prime \prime} \subset \subset V^{\prime} \subset \subset V$ a sequência $\left(\mathcal{E}_{\tau}(\chi f)\right)_{\tau=1,2, \ldots}$ é limitada em $\mathrm{G}_{\mathrm{M}}^{s, h}\left(V^{\prime \prime}\right)$ e, consequentemente, admite subsequência convergente para $f$ no espaço de Montel $\mathrm{G}_{\mathrm{M}}^{s}\left(V^{\prime \prime}\right)$.

\section{Demonstração :}

$\triangleright \quad$ Iniciamos invocando [T, II.2] para garantir que $\mathcal{E}_{\tau}(\chi f) \rightarrow \chi f \mathrm{em} \mathrm{C}^{\infty}(V)$ $\mathrm{e}$, consequentemente, garantir que

$$
\mathcal{E}_{\tau}(\chi f) \stackrel{\tau \rightarrow \infty}{\longrightarrow} f \text { em } \mathrm{C}^{\infty}\left(\overline{V^{\prime}}\right)
$$

Basta mostrarmos então que $\left(\mathcal{E}_{\tau}(f)\right)_{\tau=1,2, \ldots}$ é limitada em $\mathrm{G}_{M}^{s, h}\left(V^{\prime \prime}\right)$.

Utilizando $V^{\prime}$ para dividir o domínio de integração e usando o fato de $M^{\alpha}\left[\mathcal{E}_{\tau}(\chi f)\right]=\mathcal{E}_{\tau}\left(M^{\alpha}[\chi f]\right)$ segue que

$$
\sup _{x \in V^{\prime \prime}}\left|M^{\alpha}\left[\mathcal{E}_{\tau}(\chi f)\right](x)\right| \leq I_{1}^{\alpha}+I_{2}^{\alpha}
$$

onde

$$
\begin{aligned}
& I_{1}^{\alpha}=\sup _{x \in V^{\prime \prime}}\left(\frac{\tau}{\pi}\right)^{m / 2} \int_{x^{\prime} \in V^{\prime}} e^{-\tau \operatorname{Re}\left\langle Z(x)-Z\left(x^{\prime}\right)\right\rangle^{2}}\left|M^{\alpha}[f]\left(x^{\prime}\right)\right|\left|d Z\left(x^{\prime}\right)\right| \\
& I_{2}^{\alpha}=\sup _{x \in V^{\prime \prime}}\left(\frac{\tau}{\pi}\right)^{m / 2} \int_{x^{\prime} \notin V^{\prime}} M^{\alpha}\left[e^{-\tau \operatorname{Re}\left\langle Z(x)-Z\left(x^{\prime}\right)\right\rangle^{2}}\right]\left|(\chi f)\left(x^{\prime}\right)\right|\left|d Z\left(x^{\prime}\right)\right| .
\end{aligned}
$$


Com relação à primeira parcela, as estimativas Gevrey para $f$ garantem que

$$
I_{1}^{\alpha} \leq \mathrm{const} \cdot\|f\|_{s, h_{f}, V} \cdot h_{f}^{|\alpha|} \cdot|\alpha| !^{s}, \forall \alpha \in \mathbb{Z}_{+}^{m} .
$$

Com relação à segunda parcela, segue de (1.3) que

$$
\operatorname{Re}\left\langle Z(x)-Z\left(x^{\prime}\right)\right\rangle^{2}=\left|x-x^{\prime}\right|^{2}-\left|\Phi(x)-\Phi\left(x^{\prime}\right)\right|^{2} \geq \frac{3}{4}\left|x-x^{\prime}\right|
$$

donde a exponenciação em questão é absolutamente majorada por $e^{-d \tau}$ para alguma constante $d>0$ depende somente da distância de $V^{\prime \prime}$ ao bordo de $V^{\prime}$. Assim,

$$
I_{2}^{\alpha} \leq \text { const } \cdot \tau^{|\alpha|} e^{-\tau d}
$$

Observando que a função $\tau^{|\alpha|} e^{-\tau d}$ assume seu valor máximo em $\tau=\frac{|\alpha|}{d}$, segue que

$$
I_{2}^{\alpha} \leq \text { const } \cdot d^{-|\alpha|}|\alpha| ! \leq \text { const } \cdot h^{|\alpha|}|\alpha| !^{s} \quad, \forall \alpha \in \mathbb{Z}_{+}^{m} .
$$

Finalizamos esta seção comparando nosso Teorema 1.2 com o Teorema 5.6 em [D], a saber,

Seja $\mathcal{K} \subset \mathbb{C}^{m}$ um compacto regular e uniformemente $H$-convexo. Se $f \in \mathrm{G}^{s}(\mathcal{K}), s>1$, é a restrição de uma função $F \in \mathrm{G}^{s}\left(\mathbb{C}^{m}\right)$ com $\bar{\partial} F \sim_{\mathcal{K}} 0$ então $f$ pode ser aproximada por uma sequência $\left\{h_{j}\right\}_{j \in \mathbb{N}} \subset \mathcal{O}(\mathcal{K})$ na topologia de $\mathrm{G}^{s}(\mathcal{K})$.

O teorema [D, Teo.5.6] é caracterizado por Bernd Droste como uma versão abstrata de teoremas de aproximações holomorfas demonstrados anteriormente no artigo. Fazendo $\mathcal{K}=\Sigma$, a Proposicão 1.1 identifica as funções $f$ em [D, Teo.5.6] com funções nas classes Gevrey diferenciáveis segundo $M$ de ordem $s$, tornando tal teorema equivalente ao Teorema 1.2. No nosso teorema, as classes $G_{M}^{s}(V)$ concretizam, de certa forma, a referida abstração do teorema [D, Teo.5.6]. 


\subsection{Uma certa classe de operadores Gevrey segundo $M$}

Iniciamos esta seção introduzindo o conceito de operadores ultradiferenciáveis de tipo Beurling segundo a sequência de Gevrey $\left\{p !^{s}\right\}, s>1$, caracterizados pelas condições equivalentes da proposição a seguir envolvendo funções inteiras do tipo

$$
P(\zeta)=\sum_{|\alpha| \geq 0} a_{\alpha} \zeta^{\alpha} ;
$$

aqui $\zeta \in \mathbb{C}^{m}, \alpha \in \mathbb{Z}_{+}^{m}, a_{\alpha} \in \mathbb{C}$ e $\zeta^{\alpha}=\zeta_{1}^{\alpha_{1}} \ldots \zeta_{m}^{\alpha_{m}}$.

Proposição 1.5 Se $P(\zeta)$ é como em (1.23) e se $s>1$ então as condições abaixo são equivalentes:

$$
\begin{aligned}
\exists \varepsilon, \exists C_{\varepsilon} & :\left|a_{\alpha}\right| \leq C_{\varepsilon} \varepsilon^{|\alpha|}|\alpha| !^{-s}, \forall p \in \mathbb{Z}_{+} \\
\exists \varepsilon^{\prime}, \exists C_{\varepsilon^{\prime}} & :|P(\zeta)| \leq C_{\varepsilon^{\prime}} e^{\left(\varepsilon^{\prime}|\zeta|\right)^{1 / s}}, \forall \zeta \in \mathbb{C}^{m} .
\end{aligned}
$$

\section{Demonstração :}

$\triangleright \quad$ A equivalência entre (1.24) e (1.25) utiliza a equivalência

$$
\rho^{1 / s} \simeq N(\rho)=\ln \left(\sup _{p} \rho^{p} p !^{-s}\right) \quad, \rho>0
$$

(cf. [D, pg.297]) no sentido de existirem constantes $H_{1}, H_{2}, B_{1}, B_{2}$ tais que

$$
N\left(H_{1} \rho\right)+\ln \left(B_{1}\right) \leq \rho^{1 / s} \leq N\left(H_{2} \rho\right)+\ln \left(B_{2}\right) .
$$

Supondo (1.24) segue que

$$
\begin{aligned}
|P(\zeta)| & \leq \sum_{|\alpha| \geq 0}\left|a_{\alpha}\right|\left|\zeta_{1}\right|^{\alpha_{1}} \cdots\left|\zeta_{m}\right|^{\alpha_{m}} \leq \sum_{|\alpha| \geq 0}\left|a_{\alpha}\right||\zeta|^{|\alpha|} \\
& \leq C_{\varepsilon} \sum_{|\alpha| \geq 0}(\varepsilon|\zeta|)^{|\alpha|}|\alpha|^{-s}=C_{\varepsilon} \sum_{|\alpha| \geq 0} 2^{-|\alpha|}(2 \varepsilon|\zeta|)^{|\alpha|}|\alpha| !^{-s} \\
& \leq C_{\varepsilon} \sum_{|\alpha| \geq 0} 2^{-|\alpha|} \cdot \sup _{|\alpha|}(2 \varepsilon|\zeta|)^{|\alpha|}|\alpha| !^{-s}=C_{\varepsilon} \sum_{|\alpha| \geq 0} 2^{-|\alpha|} \cdot e^{N(2 \varepsilon|\zeta|)} \\
& \leq \frac{C_{\varepsilon} \sum^{-|\alpha|} 2^{\left.-|2 \varepsilon| \zeta \mid / H_{1}\right)^{1 / s}}}{B_{1}} e^{(2)}
\end{aligned}
$$


Por outro lado, supondo (1.25) segue pela fórmula integral de Cauchy que

$$
\begin{aligned}
\left|a_{\alpha}\right| & =\left|\partial_{\zeta}^{\alpha} P(0)\right| \leq \frac{1}{(2 \pi)^{m}} \int_{\left|\zeta_{1}\right|=\rho} \cdots \int_{\left|\zeta_{m}\right|=\rho} \frac{|P(\zeta)|}{\rho^{\alpha_{1}+1} \cdots \rho^{\alpha_{m}+1}}\left|d \zeta_{1}\right| \ldots\left|d \zeta_{m}\right| \\
& \leq \frac{C_{\varepsilon^{\prime}}}{(2 \pi)^{m}} \int_{\left|\zeta_{1}\right|=\rho} \cdots \int_{\left|\zeta_{m}\right|=\rho} \frac{e^{\left(\varepsilon^{\prime}|\zeta|\right)^{1 / s}}}{\rho^{\alpha_{1}+1} \cdots \rho^{\alpha_{m}+1}}\left|d \zeta_{1}\right| \ldots\left|d \zeta_{m}\right| \\
& \leq C_{\varepsilon^{\prime}} e^{\left(\sqrt{m} \varepsilon^{\prime} \rho\right)^{1 / s}} \rho^{-|\alpha|} \leq C_{\varepsilon^{\prime}} B_{2} e^{N\left(H_{2} \sqrt{m} \varepsilon^{\prime} \rho\right)} \rho^{-|\alpha|} \\
& =C_{\varepsilon^{\prime}} B_{2}\left(H_{2} \sqrt{m} \varepsilon^{\prime}\right)^{|\alpha|} \sup _{p}\left(H_{2} \sqrt{m} \varepsilon^{\prime} \rho\right)^{p-|\alpha|} p !^{-s}, \forall \rho>0, \\
& \leq C_{\varepsilon^{\prime}} B_{2}\left(H_{2} \sqrt{m} \varepsilon^{\prime}\right)^{|\alpha|} \cdot \inf _{\rho} \sup _{p} \rho^{p-|\alpha|} p !^{-s} \\
& =C_{\varepsilon^{\prime}} B_{2}\left(H_{2} \sqrt{m} \varepsilon^{\prime}\right)^{|\alpha|}|\alpha| !^{-s} .
\end{aligned}
$$

Registramos para uso futuro que

$$
\varepsilon=H_{2} \sqrt{m} \cdot \varepsilon^{\prime} \text { e } C_{\varepsilon}=B_{2} \cdot C_{\varepsilon^{\prime}} .
$$

Definição 1.5 Dizemos que um operador $P=P(\mathrm{M})$ do tipo

$$
P(\mathrm{M})=\sum_{|\alpha| \geq 0} a_{\alpha}(-i \mathrm{M})^{\alpha}, a_{\alpha} \in \mathbb{C}
$$

é Gevrey diferencial segundo $\mathrm{M}$ de ordem $s>1$ com símbolo dado por (1.23) quando seus coeficientes $a_{\alpha}$ satisfazem a condição (1.24) ou, equivalentemente, quando seu símbolo $P(\zeta)$ satisfaz a condição (1.25).

É importante ressaltar que os operadores Gevrey diferenciais segundo M acima definidos não estabelecem, a priori, endomorfismos nas classes Gevrey diferenciáveis segundo $M$ abordadas nesta tese (diferenciabilidade Gevrey de tipo Roumieu). Na verdade tais operadores definem endomorfismos em classes Gevrey diferenciáveis segundo $M$ definidas a partir da existência, na estimativa (1.8), de uma constante universal em relação às amplitudes $h>0$ (diferenciabilidade Gevrey de tipo Beurling). Para (1.28) determinar endomorfismos nas classes $\mathrm{G}_{\mathrm{M}}^{s}(U)$ basta trocar em (1.24) [resp.(1.25)] o quantificador $\exists \varepsilon$ por $\forall \varepsilon$ [resp. $\exists \varepsilon^{\prime}$ por $\left.\forall \varepsilon^{\prime}\right]$. 
Vamos trabalhar nesta tese com certas classes de operadores Gevrey diferenciais segundo $\mathrm{M}$, denominadas classe de Kaneko de ordem $s>1$, onde os símbolos se expressam na forma

$$
P_{r}(\zeta)=\prod_{p=1}^{\infty}\left(1+\frac{r^{2}}{p^{2 s}}\langle\zeta\rangle^{2}\right), 0<r<1
$$

como lembrete, $\langle\zeta\rangle^{2}=\zeta_{1}^{2}+\cdots+\zeta_{m}^{2}$. Façamos um parênteses para observar que a classe de Kaneko considerada por Cordaro em [C] é construída a partir de símbolos

$$
P(\zeta)=\prod_{p=1}^{\infty}\left(1+\frac{\langle\zeta\rangle^{2}}{p^{2}(\sigma(p-1))^{2}}\right)
$$

satisfazendo estimativas exponenciais do tipo

$$
\begin{gathered}
\forall \epsilon, \exists C_{\epsilon}:|P(\zeta)| \leq C_{\epsilon} e^{\epsilon|\zeta|}, \forall \zeta \in \mathbb{C}^{m} \\
\exists \rho \in] 0,1\left[, \exists C_{\rho}, \delta_{\rho}:|P(\zeta)| \geq C_{\rho} \exp \left\{\delta_{\rho} \frac{|\zeta|}{\sigma(|\zeta|)}\right\}, \forall \zeta \in \Gamma_{\rho}\right.
\end{gathered}
$$

onde $\sigma:[0, \infty] \rightarrow \mathbb{R}$ é uma função monótona crescente ao infinito com $\sigma(0)=1$ e $\Gamma_{\rho}$ é o cone complexo $|\operatorname{Im} \zeta| \leq \rho|\operatorname{Re} \zeta|$. As classes de Kaneko de ordem $s>1$ são construídas a partir das funções

$$
\sigma_{r}(t)=\left(\frac{(t+1)^{s-1}}{r}\right)^{2} .
$$

Iniciamos o estudo de nossas classes de Kaneko estabelecendo as estimativas exponenciais para seus elementos. No que segue, $P_{r}(\mathrm{M})$ denota um operador qualquer numa fixada classe de Kaneko de ordem $s>1$.

Lema 1 Os operadores $P_{r}(\mathrm{M})$ satisfazem as estimativas (1.25) e (1.24) em respectivos $C_{\varepsilon^{\prime}}=1, \varepsilon^{\prime}=H r$ e $C_{\varepsilon}=B_{2}, \varepsilon=H_{2} \sqrt{m} H r$, onde $H_{2}, B_{2}$ advém de $(1.26)$ e $H=\left(32^{s} s^{2 s}\right)$. Mais ainda, $P_{r}(\mathrm{M})$ também satisfaz a estimativa infra-exponencial (1.30).

\section{Demonstração :}

$\triangleright$ Observe inicialmente que

$$
\left|P_{r}(\zeta)\right| \leq \prod_{p=1}^{\infty}\left(1+\frac{r^{2}}{p^{2 s}}\left(\left|\zeta_{1}^{2}\right|+\cdots+\left|\zeta_{m}^{2}\right|\right)\right)=\prod_{p=1}^{\infty}\left(1+\frac{r^{2}|\zeta|^{2}}{p^{2 s}}\right) .
$$


Aplicando logaritmo no produtório obtemos

$$
\sum_{p=1}^{\infty} \ln \left(1+\frac{r^{2}|\zeta|^{2}}{p^{2 s}}\right) \leq \int_{0}^{\infty} \ln \left(1+\frac{r^{2}|\zeta|^{2}}{\eta^{2 s}}\right) d \eta
$$

Integrando duas vezes por partes, as contribuições da fronteira

$$
\left[\eta \ln \left(1+r^{2}|\zeta|^{2} / \eta^{2 s}\right)+2 s \eta r^{2}|\zeta|^{2} /\left(\eta^{2 s}+r^{2}|\zeta|^{2}\right)\right]_{\eta \rightarrow 0}^{\eta \rightarrow \infty}
$$

se anulam e a integral resultante será

$$
(4 s)^{2} \int_{0}^{\infty} \frac{r^{2}|\zeta|^{2} \eta^{2 s}}{\left(\eta^{2 s}+r^{2}|\zeta|^{2}\right)^{2}} d \eta
$$

Dividindo o intervalo de integração em $(r|\zeta|)^{1 / s}$ obtemos

$$
\begin{aligned}
\int_{0}^{(r|\zeta|)^{1 / s}} \frac{r^{2}|\zeta|^{2} \eta^{2 s}}{\left(\eta^{2 s}+r^{2}|\zeta|^{2}\right)^{2}} d \eta & \leq \int_{0}^{(r|\zeta|)^{1 / s}} \frac{\left(r^{2}|\zeta|^{2}\right)^{2}}{\left(\eta^{2 s}+r^{2}|\zeta|^{2}\right)^{2}} d \eta \leq(r|\zeta|)^{1 / s} \\
\int_{(r|\zeta|)^{1 / s}}^{\infty} \frac{r^{2}|\zeta|^{2} \eta^{2 s}}{\left(\eta^{2 s}+r^{2}|\zeta|^{2}\right)^{2}} d \eta & \leq r^{2}|\zeta|^{2} \int_{(r|\zeta|)^{1 / s}}^{\infty} \eta^{-2 s} d \eta \leq(r|\zeta|)^{1 / s}
\end{aligned}
$$

Assim

$$
(4 s)^{2} \int_{0}^{\infty} \frac{r^{2}|\zeta|^{2} \eta^{2 s}}{\left(\eta^{2 s}+r^{2}|\zeta|^{2}\right)^{2}} d \eta \leq 32 s^{2}(r|\zeta|)^{1 / s}=\left(32^{s} s^{2 s} r|\zeta|\right)^{1 / s}
$$

e

$$
\left|P_{r}(\zeta)\right| \leq e^{\int_{0}^{\infty} \ln \left(1+\frac{r^{2}|\zeta|^{2}}{\eta^{2 s}}\right) d \eta} \leq e^{\left(32^{s} s^{2 s} r|\zeta|\right)^{1 / s}} .
$$

Portanto existe $\varepsilon^{\prime}=\left(32^{s} s^{2 s}\right) r$ e $C_{\varepsilon^{\prime}}=1$ cumprindo (1.25).

A existência de $\varepsilon=H_{2} \sqrt{m}\left(32^{s} s^{2 s}\right) r$ e $C_{\varepsilon}=B_{2}$ cumprindo (1.24) é uma consequência imediata de (1.27).

Com relação à (1.30), como existe $\delta=32 s^{2} r^{1 / s}$ tal que

$$
\left|P_{r}(\zeta)\right| \leq e^{\delta|\zeta|^{1 / s}}, \forall \zeta \in \mathbb{C}^{m}
$$

segue, para $\epsilon>0$ fixado, que

$$
\begin{aligned}
& |\zeta| \leq(\delta / \epsilon)^{\frac{s-1}{s}} \Rightarrow\left|P_{r}(\zeta)\right| \leq e^{\delta|\zeta|^{1 / s}} \leq e^{\delta(\delta / \epsilon)^{\frac{s-1}{s^{2}}}}=C_{\epsilon} \leq C_{\epsilon} e^{\epsilon|\zeta|} \\
& |\zeta|>(\delta / \epsilon)^{\frac{s-1}{s}} \Rightarrow \epsilon|\zeta|>\delta|\zeta|^{1 / s} \Rightarrow\left|P_{r}(\zeta)\right| \leq e^{\delta|\zeta|^{1 / s}} \leq e^{\epsilon|\zeta|},
\end{aligned}
$$

concluíndo assim a demonstração. 
Lema 2 Para todo $\varrho \in] 0,1\left[\right.$ e para todo $\theta \in \mathbb{C}^{m} \operatorname{com}|\operatorname{Im} \theta|<\varrho|\operatorname{Re} \theta|$, valem as estimativas supra-exponenciais

$$
\begin{aligned}
& \left|P_{r}(\theta)\right| \geq|\theta|^{p}(\underline{\tilde{o}} r)^{p} p !^{-s}, \forall p \in \mathbb{Z}_{+} \\
& \left|P_{r}(\theta)\right| \geq \frac{1}{B_{2}} \exp \left\{\left(\tilde{\varrho} / H_{2}\right)^{1 / s} \frac{|\theta|}{\sigma_{r}(|\theta|+1)}\right\}
\end{aligned}
$$

onde $\tilde{\varrho}=\sqrt{\frac{1-\varrho^{2}}{1+\varrho^{2}}}$ e $H_{2}, B_{2}$ advém de (1.26).

\section{Demonstração :}

$\triangleright \quad$ Observe que no cone em questão

$$
\begin{aligned}
\operatorname{Re}\langle\theta\rangle^{2} & =|\operatorname{Re} \theta|^{2}-|\operatorname{Im} \theta|^{2} \geq\left(1-\varrho^{2}\right)|\operatorname{Re} \theta|^{2}=\tilde{\varrho}^{2}\left(1+\varrho^{2}\right)|\operatorname{Re} \theta|^{2} \\
& \geq \tilde{\varrho}^{2}\left(|\operatorname{Re} \theta|^{2}+|\operatorname{Im} \theta|^{2}\right)=\tilde{\varrho}^{2}|\theta|^{2} .
\end{aligned}
$$

A estimativa (1.33) segue de

$$
\begin{aligned}
|P(\theta)| & =\sup _{p} \prod_{q=1}^{p}\left|1+\frac{r^{2}}{q^{2 s}}\langle\theta\rangle^{2}\right| \geq \sup _{p} \prod_{q=1}^{p}\left(1+\frac{r^{2}}{q^{2 s}} \operatorname{Re}\langle\theta\rangle^{2}\right) \\
& \geq \sup _{p} \prod_{q=1}^{p}\left(1+\frac{\tilde{\varrho}^{2} r^{2}|\theta|^{2}}{q^{2 s}}\right) \geq \sup _{p} \prod_{q=1}^{p} \frac{\tilde{\varrho} r|\theta|}{q^{s}} \\
& =\sup _{p}(\tilde{\varrho} r|\theta|)^{p} p !^{-s} .
\end{aligned}
$$

A estimativa (1.34) segue de (1.33) e (1.26), ou seja,

$$
\begin{aligned}
|P(\theta)| & \geq \sup _{p}(\underline{\varrho} r|\theta|)^{p} p !^{-s}=e^{N(\tilde{\varrho} r|\theta|)} \\
& \geq \frac{1}{B_{2}} \exp \left\{\left(\tilde{\varrho} r / H_{2}\right)^{1 / s}|\theta|^{1 / s}\right\} \\
& =\frac{1}{B_{2}} \exp \left\{\left(\tilde{\varrho} / H_{2}\right)^{1 / s} \frac{|\theta|}{\left(|\theta|^{s-1} / r\right)^{1 / s}}\right\} \\
& \geq \frac{1}{B_{2}} \exp \left\{\left(\tilde{\varrho} / H_{2}\right)^{1 / s} \frac{|\theta|}{\left((|\theta|+1)^{s-1} / r\right)^{2}}\right\} \\
& \geq \frac{1}{B_{2}} \exp \left\{\left(\tilde{\varrho} / H_{2}\right)^{1 / s} \frac{|\theta|}{\sigma(|\theta|+1)}\right\} .
\end{aligned}
$$


Neste ponto vamos invocar $[\mathrm{C}]$. Os operadores $P_{r}\left(\partial_{z}\right)$ definidos como em (1.28) através da base $\partial_{z}=\left(\frac{\partial}{\partial z_{1}}, \ldots, \frac{\partial}{\partial z_{m}}\right)$ determinam, via estimativa (1.30), endomorfismos nos espaços $\mathcal{O}(\mathcal{U})$ das funções holomorfas em $\mathcal{U} \subset \mathbb{C}^{m}$. Em particular, $P_{r}\left(\partial_{z}\right)\left[e^{i \zeta \cdot z}\right]=P_{r}(\zeta) e^{i \zeta \cdot z} \mathrm{e}$

$$
P_{r}(\mathrm{M})\left[e^{i \zeta \cdot Z(x, t)}\right]=P_{r}(\zeta) e^{i \zeta \cdot Z(x, t)}, \forall \zeta \in \mathbb{C}^{m} .
$$

Vamos trabalhar também numa pequena vizinhança $V \subset \subset B$ da origem e com números reais $\kappa, \delta_{0}>0$ pequenos para validar as estimativas

$$
\begin{aligned}
& \operatorname{Im}\left[\zeta \cdot\left(Z-Z^{\prime}-i v\right)+i\langle\zeta\rangle\left\langle Z-Z^{\prime}-i v\right\rangle^{2}\right] \geq(1-\kappa)\left|Z-Z^{\prime}\right|^{2}|\zeta|-3|v||\zeta| \\
& |\operatorname{Im} \theta|<\frac{1}{2 \sqrt{3}}|\operatorname{Re} \theta| \quad, \quad \frac{|\zeta|}{2} \leq|\theta| \leq 3|\zeta| \quad, \quad \operatorname{Re}\langle\theta\rangle^{2} \geq \frac{|\zeta|^{2}}{2}
\end{aligned}
$$

em quaisquer

- $Z, Z^{\prime} \in Z(v) \operatorname{com} Z=Z(x)$ e $Z^{\prime}=Z\left(x^{\prime}\right)$,

- $\zeta \in \mathbb{R}^{\prime}{ }_{Z^{\prime}} \Sigma=\left\{\zeta \cdot d Z^{\prime}: \zeta={ }^{t} Z_{x}^{-1}\left(x^{\prime}\right) \cdot \xi, \xi \in \mathbb{R}^{m}\right\}$,

- $v \in \mathbb{R}^{m} \operatorname{com}|v| \leq 1$,

- $\theta=\zeta+i\left(Z-Z^{\prime}+i y\right)\langle\zeta\rangle$ onde $y \in \mathbb{R}^{m}$ e tal que $|y|<\delta_{0}$; aqui a função $\langle\zeta\rangle=\left(\langle\zeta\rangle^{2}\right)^{1 / 2}$ é bem definida já que $|\operatorname{Im} \zeta|<|\operatorname{Re} \zeta|$.

O contexto acima (cf. [C, (1.12)-1.15)]) é fundamental para os dois lemas a seguir.

Lema 3 Fixado $r \in] 0,1\left[\right.$ existe $h_{r}>0$ tal que qualquer função $v \in \mathrm{C}^{\infty}(V)$ é representada numa vizinhança $V^{\prime} \subset \subset V$ da origem por $v=P_{r}(\mathrm{M}) w$ onde $w \in \mathrm{G}_{\mathrm{M}}^{s, h_{r}}\left(V^{\prime}\right)$.

\section{Demonstração :}

$\triangleright \quad$ Iniciamos introduzindo uma função de corte $\chi$ suportada em $V$ com $\chi \equiv 1$ em $V^{\prime}$.

Fixada $v \in \mathbb{C}^{\infty}(V)$ vamos definir, para cada $\epsilon>0$, funções $v_{\epsilon}: V \rightarrow \mathbb{C}$ através das representações integrais

$$
v_{\epsilon}(x)=\frac{1}{(2 \pi)^{m}} \int_{x^{\prime} \in \mathbb{R}^{m}} \int_{\xi \in \mathbb{R}^{m}} \frac{e^{i \xi \cdot\left(Z(x)-Z\left(x^{\prime}\right)\right)-\epsilon\langle\xi\rangle^{2}}}{P_{r}(\xi)}[\chi v]\left(x^{\prime}\right) \operatorname{det} Z_{x}\left(x^{\prime}\right) \mathrm{d} \xi \mathrm{d} x^{\prime} .
$$


Desnecessário observar que a estimativa supra-exponencial (1.34) justifica a presença de $P_{r}(\xi)$ no denominador.

Utilizando (1.35) e integrando em $\xi$ é possível expressar

$$
\begin{aligned}
P_{r} v_{\epsilon}(x) & =\frac{1}{(2 \pi)^{m}} \iint e^{i \zeta \cdot\left(Z(x)-Z\left(x^{\prime}\right)\right)-\epsilon\langle\zeta\rangle^{2}}[\chi v]\left(x^{\prime}\right) \operatorname{det} Z_{x}\left(x^{\prime}\right) \mathrm{d} \xi \mathrm{d} x^{\prime} \\
& =\frac{1}{(4 \pi \epsilon)^{m / 2}} \int e^{-\frac{1}{4 \epsilon}\left\langle Z(x)-Z\left(x^{\prime}\right)\right\rangle^{2}}[\chi v]\left(x^{\prime}\right) \operatorname{det} Z_{x}\left(x^{\prime}\right) \mathrm{d} x^{\prime}
\end{aligned}
$$

através de gaussianas que convergem uniformemente para $\chi v$ em compactos de $V$ (cf. [T, Sec.II.2]), donde

$$
P_{r} v_{\epsilon} \stackrel{\epsilon \rightarrow 0}{\longrightarrow} v \text { uniformemente em } \overline{V^{\prime}} .
$$

Por outro lado, a presença de $e^{-i \xi \cdot Z_{k}^{\prime}}$ no integrando de $v_{\epsilon}$ permite, após integração por partes em $x^{\prime}{ }_{k}$, introduzir o fator $\left(1+\left|\xi_{k}\right|^{2}\right)$ no denominador pagando-se o preço de substituir $\chi v$ por $\left(1-\mathrm{M}_{k}^{2}\right)[\chi v]$. Mais ainda, após uma deformação do tipo $\xi \mapsto \zeta \mapsto \theta=\zeta+i\left(Z-Z^{\prime}\right)\langle\zeta\rangle$, vamos obter a seguinte expressão para $\mathrm{M}^{\alpha} v_{\epsilon}$, a saber,

$$
\frac{1}{(2 \pi)^{m}} \int_{Z^{\prime} \in \Sigma} \int_{\zeta \in \mathbb{R} T_{Z^{\prime}}^{\prime} \Sigma} \frac{(i \theta)^{\alpha} e^{i \theta \cdot\left(Z-Z^{\prime}\right)-\epsilon\langle\theta\rangle^{2}}}{P_{r}(\theta) \prod\left(1+\left|\theta_{k}\right|^{2}\right)} \prod\left(1-\mathrm{M}_{k}^{2}\right)[\chi v] \Delta\left(Z-Z^{\prime}, \zeta\right) \mathrm{d} \zeta \mathrm{d} Z^{\prime}
$$

onde $\Delta\left(Z-Z^{\prime}, \zeta\right)$ denota o determinante Jacobiano da aplicação $\zeta \mapsto \theta$. $\mathrm{O}$ primeiro fato importante é que (1.36) garante, via estimativa (1.33) do Lema 2, que

$$
\left|P_{r}(\theta)\right| \geq|\theta|^{|\alpha|}\left(\sqrt{\frac{11}{13}} r\right)^{-|\alpha|}|\alpha|^{!^{-s}}, \forall \alpha \in \mathbb{Z}_{+}^{m} .
$$

O segundo fato importante é que (1.36) garante que

$$
\left|e^{i \theta \cdot\left(Z-Z^{\prime}\right)-\epsilon\langle\theta\rangle^{2}}\right|=e^{-\operatorname{Im}\left[\zeta \cdot\left(Z-Z^{\prime}\right)+i\langle\zeta\rangle\left\langle Z-Z^{\prime}\right\rangle^{2}\right]-\epsilon \operatorname{Re}\langle\theta\rangle^{2}} \leq 1 .
$$

Isto posto, o integrando de $\mathrm{M}^{\alpha} v_{\epsilon}$ é absolutamente e uniformemente majorado por

$$
\text { const } \cdot\left(\sqrt{\frac{11}{13}} r\right)^{-|\alpha|}|\alpha| !^{s}\left(1+|\theta|^{2}\right)^{-m}
$$


onde a constante independe de $\theta$ e de $\alpha$. O teorema da convergência dominada garante a existência de

$$
\lim _{\epsilon \rightarrow 0} v_{\epsilon}=w \in \mathrm{G}_{\mathrm{M}}^{s, h_{r}}\left(V^{\prime}\right), h_{r}=\left(\sqrt{\frac{11}{13}} r\right)^{-1},
$$

e a unicidade do limite garante que

$$
P_{r}(\mathrm{M}) w=v \quad \text { em } \quad V^{\prime} .
$$

O próximo lema é uma aplicação direta do Teorema 1.2 em [C].

Lema 4 Fixada qualquer vizinhança $V^{\prime} \subset \subset V$ da origem sempre existe uma vizinhança $\mathcal{U} \subset \mathbb{C}^{m}$ de $Z\left(V^{\prime}\right)$ na qual as soluções $g \in \mathrm{G}_{\mathrm{M}}^{s, h}(V)$ de $P_{r}(\mathrm{M}) g=0$ se estendem holomorficamente, isto é, existe $G=G(z)$ holomorfa em $\mathcal{U}$ com $\left.G \circ Z\right|_{V^{\prime}}=\left.g\right|_{V^{\prime}}$.

Finalizamos esta seção estabelecendo a atuação de certos operadores na classe de Kaneko de ordem $s>1$ nos espaços $\mathrm{G}_{\mathrm{M}}^{s, h}(V)$.

Lema 5 Fixada $h>0$ existe $r_{h}>0$ tal que, para todo $0<r \leq r_{h}$,

$$
P_{r}(\mathrm{M}): \mathrm{G}_{\mathrm{M}}^{s, h}(V) \rightarrow \mathrm{G}_{\mathrm{M}}^{s, h^{+}}(V) \quad, \forall h^{+}>h .
$$

\section{Demonstração :}

$\triangleright \quad$ Pelo Lema 1 podemos expressar $P_{r}(\mathrm{M})$ na forma $\sum a_{\alpha^{\prime}} \mathrm{M}^{\alpha^{\prime}}$ com $\left|a_{\alpha^{\prime}}\right|$ satisfazendo (1.24) para $\varepsilon=H_{2} \sqrt{m} H r$ e $C_{\varepsilon}=B_{2}$.

Fixada $f \in \mathrm{G}_{\mathrm{M}}^{s, h}(V)$ as parcelas $a_{\alpha^{\prime}} \mathrm{M}^{\alpha^{\prime}} f$ de $P_{r}(\mathrm{M}) f$ satisfazem

$$
\begin{aligned}
\sup _{\bar{V}}\left|\mathrm{M}^{\alpha}\left[a_{\alpha^{\prime}} \mathrm{M}^{\alpha^{\prime}} f\right]\right| & =\left|a_{\alpha^{\prime}}\right| \sup _{\bar{V}}\left|\mathrm{M}^{\alpha+\alpha^{\prime}} f\right| \\
& \leq B_{2}|| f \|_{s, h, V}\left(H_{2} \sqrt{m} H h r\right)^{\left|\alpha^{\prime}\right|} h^{|\alpha|} \frac{\left(|\alpha|+\left|\alpha^{\prime}\right|\right) !^{s}}{\left|\alpha^{\prime}\right| ! s} .
\end{aligned}
$$

Usando a estabilidade da sequência $\left\{p !^{s}\right\}$ sobre operadores Gevreydiferenciais, a saber,

$$
\forall \epsilon \exists C_{\epsilon}: p !^{s} \leq C_{\epsilon}(1+\epsilon)^{p} \min _{0 \leq q \leq p} q !^{s}(p-q) !^{s}, \forall p
$$


$\operatorname{com} p=\left|\alpha^{\prime}\right|+|\alpha|$ e $q=\left|\alpha^{\prime}\right|$, vamos obter

$\sup _{\bar{V}}\left|\mathrm{M}^{\alpha}\left[a_{\alpha^{\prime}} \mathrm{M}^{\alpha^{\prime}} f\right]\right| \leq B_{2}\|f\|_{s, h, V} C_{\epsilon}\left((1+\epsilon) H_{2} \sqrt{m} H h r\right)^{\left|\alpha^{\prime}\right|}((1+\epsilon) h)^{|\alpha|}|\alpha| !^{s}$.

O lema segue tomando $r_{h}$ pequeno para tornar

$$
\left((1+\epsilon) H_{2} \sqrt{m} H h r_{h}\right)^{\left|\alpha^{\prime}\right|}
$$

somável em $\alpha^{\prime}$. 


\section{Capítulo 2}

\section{Soluções Gevrey hipo-analíticas}

Este capítulo trata de questões envolvendo a diferenciabilidade Gevrey de ordem $s>1$ segundo estruturas hipo-analíticas de codimensão $n$ numa variedade diferenciável $(m+n)$-dimensional. O espaço das soluções Gevrey segundo a estrutura é introduzido e o principal resultado estabelece o teorema de Baouendi-Treves para tais soluções.

\subsection{Estruturas hipo-analíticas com codimen- são: a base $(M, L)$}

Seja $\mathcal{M}$ uma variedade diferenciável $(m+n)$-dimensional, $m, n \geq 1$, equipada com uma estrutura hipo-analítica de codimensão $n$. Tal estrutura é caracterizada, como no capítulo anterior, pela existência de uma coleção de cartas hipo-analíticas $\left(\Omega_{\imath}, Z_{\imath}\right)$ onde $\Omega_{\imath}$ são abertos cobrindo $\mathcal{M}$ e $Z_{\imath}=$ $\left(Z_{\imath 1}, \ldots, Z_{\imath m}\right)$ são aplicações infinitamente diferenciáveis em $\Omega_{\imath}$, a valores em $\mathbb{C}^{m}$, satisfazendo

- para cada $\imath$, as diferenciais $\mathrm{d} Z_{\imath 1}, \ldots, \mathrm{d} Z_{\imath m}$ são $\mathbb{C}$-linearmente independentes em cada ponto de $\Omega_{i}$;

- para cada par $\imath \neq \kappa$ e para cada $P \in \Omega_{\imath} \cap \Omega_{\kappa}$, existe um biholomorfismo $H$ de uma vizinhança aberta de $Z_{\imath}(P) \subset \mathbb{C}^{m}$ numa vizinhança aberta de $Z_{\kappa}(P) \subset \mathbb{C}^{m}$ tal que $Z_{\kappa}=H \circ Z_{\imath}$ numa vizinhança de $P$.

Fixada uma origem $O$ qualquer na variedade vamos trabalhar, via biholomorfismos que preservam a origem e [T, Sec.II.1], numa situável carta hipo- 
analítica $(\Omega, Z)$, onde $\Omega$ é um domínio local de coordenadas

$$
(x, t)=\left(x_{1}, \ldots, x_{m}, t_{1}, \ldots, t_{n}\right)
$$

centrado na origem nas quais

$$
Z_{k}(x, t)=x_{k}+i \Phi_{k}(x, t), k=1, \ldots, m
$$

com $\Phi=\left(\Phi_{1}, \ldots, \Phi_{m}\right)$ infinitamente diferenciável em $\Omega$, a valores em $\mathbb{R}^{m}$, satisfazendo

$$
|\Phi(x, t)| \leq \text { const } \cdot\left(|x|^{3}+|t|\right) .
$$

Vamos assumir

$$
\Omega=B \times \Theta \ni(x, t),
$$

onde $B$ e $\Theta$ são pequenas bolas abertas centradas nas respectivas origens do $x$-espaço $\mathbb{R}^{m}$ e do $t$-espaço $\mathbb{R}^{n}$, tornando a aplicação

$$
\lambda: \Omega \rightarrow \mathbb{C}^{m} \times \mathbb{R}^{n}, \quad(x, t) \stackrel{\lambda}{\mapsto}(Z(x, t), t)
$$

um difeomorfismo entre $\Omega$ e a subvariedade

$$
\Sigma=\{(Z(x, t), t):(x, t) \in \Omega\} .
$$

A pequenez de $B$ e $\Theta$ torna as aplicações $x \mapsto Z(x, t)$ difeomorfismos entre $B \times\{t\}$ e as subvariedades reais maximais de $\mathbb{C}^{m}$ dadas por

$$
\Sigma_{t}=\{Z(x, t): x \in B\} .
$$

Destacamos a base de $\mathbb{C T} \mathcal{M}$ sobre $\Omega$ dual de $\left(d Z_{1}, \ldots, d Z_{m}, d t_{1}, \ldots, d t_{n}\right)$, a saber,

$$
(M, L)=\left(M_{1}, \ldots, M_{m}, L_{1}, \ldots, L_{n}\right)
$$

onde $\mathrm{M}_{k}$ e $\mathrm{L}_{j}$ são tais que

$$
\mathrm{M}_{k} t_{j}=0, \mathrm{~L}_{j} Z_{k}=0, \mathrm{M}_{k} Z_{k^{\prime}}=\delta_{k k^{\prime}}, \mathrm{L}_{j} t_{j^{\prime}}=\delta_{j j^{\prime}} .
$$

Aqui $\delta$ é a função delta de Kronecker. 


\subsection{Diferenciabilidade Gevrey segundo $(M, L)$}

O conceito de diferenciabilidade Gevrey segundo ( $\mathrm{M}, \mathrm{L})$ de ordem $s \geq 1$, no fecho de uma vizinhança $U \subset \Omega$ da origem e numa amplitude $h>0$, advém da estimativa

$$
\sup _{\bar{U}}\left|M^{\alpha} L^{\gamma} f\right| \leq \text { const } \cdot h^{|\alpha|+|\gamma|}(|\alpha|+|\gamma|) !^{s}, \forall(\alpha, \gamma) \in \mathbb{Z}_{+}^{m+n} .
$$

Tal conceito equivale ao conceito usual de ultradiferenciabilidade com respeito à sequência não quase analítica $\left\{p^{!^{s}}\right\}$ para o caso Roumieu (cf. [K]) exceto por utilizar em $(2.10)$ a base $(M, L)=\left(M_{1}, \ldots, M_{m}, L_{1}, \ldots, L_{n}\right)$ ao invés da base usual $\mathrm{D}=\left(\frac{\partial}{\partial x_{1}}, \ldots, \frac{\partial}{\partial x_{m}}, \frac{\partial}{\partial t_{1}}, \ldots, \frac{\partial}{\partial t_{n}}\right)$.

Definição 2.1 Dizemos que uma função $f$ infinitamente diverenciável no fecho de uma vizinhança $U \subset \subset \Omega$ da origem é de classe s-Gevrey segundo $(\mathrm{M}, \mathrm{L})$ em $U$ numa amplitude $h>0$ quando $f$ cumpre a estimativa (2.10) para alguma constante positiva. O subespaço de $\mathrm{C}^{\infty}(\bar{U})$ constituído por tais funções será denotado por $\mathrm{G}_{\mathrm{ML}}^{s, h}(U)$.

Como no capítulo anterior, cada $\mathrm{G}_{\mathrm{ML}}^{s, h}(U)$ é um espaço de Banach em relação à norma definida pelo ínfimo das constantes em (2.10), a saber,

$$
\|f\|_{s, h, U}=\sup \left\{\frac{\left|\mathrm{M}^{\alpha} L^{\gamma} f(x)\right|}{h^{|\alpha|+|\gamma|}(|\alpha|+|\gamma|) !^{s}}:(x, t) \in \bar{U},(\alpha, \gamma) \in \mathbb{Z}_{+}^{m+n}\right\}
$$

e as inclusões

$$
\mathrm{G}_{\mathrm{ML}}^{s, h}(U) \hookrightarrow \mathrm{G}_{\mathrm{ML}}^{s, h_{+}}(U), h<h_{+}
$$

também são compactas. Só para evidenciar,

$$
\mathrm{G}_{\mathrm{ML}}^{s, h}(U)=\left\{f \in \mathrm{C}^{\infty}(\bar{U}):\|f\|_{s, h, U}<\infty\right\} .
$$

Definição 2.2 Dizemos que uma função $f$ é de classe $s$-Gevrey segundo $(\mathrm{M}, \mathrm{L})$ numa vizinhança $U \subset \subset \Omega$ da origem quando $f$ pertence a $\mathrm{G}_{\mathrm{ML}}^{s, h}(U)$ para alguma amplitude $h>0$. O subespaço de $C^{\infty}(\bar{U})$ constituído por tais funções será denotado por $\mathrm{G}_{\mathrm{ML}}^{s}(U)$. 
Também como no capítulo anterior, os espaços

$$
\mathrm{G}_{\mathrm{ML}}^{s}(U)=\underset{h \lim _{\infty}}{\underset{\mathrm{ML}}{\longrightarrow}} \mathrm{G}_{\mathrm{ML}}^{s, h}(U)
$$

são Hausdorff e Montel com a topologia de limite indutivo. Em particular, sequências limitadas em algum $\mathrm{G}_{\mathrm{ML}}^{s, h}(U)$ admitem subsequências convergentes em $\mathrm{G}_{\mathrm{ML}}^{s, h_{+}}(U)$ para todo $h_{+}>h$ e, consequentemente, em $\mathrm{G}_{\mathrm{ML}}^{s}(U)$.

Vamos denotar por $\mathrm{G}_{\mathrm{ML}}^{s}(0)$ o espaço dos germes na origem de funções Gevrey segundo $(M, L)$, caracterizados pela relação de equivalência que identifica duas funções Gevrey segundo (M, L) coincidentes no fecho de alguma vizinhança da origem. Só para evidenciar,

$$
\mathrm{G}_{\mathrm{ML}}^{s}(0)=\left\{\text { classes } \mathbf{f} \text { representadas por } f \in \mathrm{G}_{\mathrm{ML}}^{s}(U), 0 \in U \subset \subset \Omega\right\} \text {. }
$$

Segue como no capítulo anterior as seguintes observações:

Observação 1: as classes de funções Gevrey usuais, denotadas nesta tese por $\mathrm{G}^{s, h}(U), \mathrm{G}^{s}(U)$ e $\mathrm{G}^{s}(0)$, são aquelas onde $(\mathrm{M}, \mathrm{L})=\mathrm{D}$ ou, equivalentemente, $Z(x, t)=x$.

Observação 2: as classes de funções Gevrey segundo $(\mathrm{M}, \mathrm{L})$ de ordem $s$ coincidem com as classes de funções Gevrey usuais de ordem $s$ no caso em que as componentes $Z_{1}, \ldots, Z_{m}$ da carta hipo-analítica $(\Omega, Z)$ são Gevrey usuais de ordem $s$ em $\Omega$.

\subsection{Funções Gevrey hipo-analíticas}

Como no caso $\mathrm{C}^{\infty}$, o conceito de soluções Gevrey hipo-analíticas com respeito à estrutura gerada por $\mathrm{d} Z_{1}, \ldots, \mathrm{d} Z_{m}$ é obtido através das soluções do sistema homogêneo $L_{j} h=0$.

Definição 2.3 Dizemos que uma função $h \in \mathrm{G}_{\mathrm{ML}}^{s}(U)$ é uma função $s$-Gevrey hipo-analítica quando $\mathrm{L}_{j} h=0$ em $U$ para todo $j=1, \ldots, n$. O subespaço fechado de $\mathrm{G}_{\mathrm{ML}}^{s}(U)$ constituído por tais funções será denotado por $\mathrm{G}_{h a}^{s}(U)$ e o espaço dos germes na origem de tais funções será denotado por $\mathrm{G}_{h a}^{s}(0)$.

Da mesma forma que as funções Gevrey diferenciáveis segundo $M$ são caracterizadas em $\mathbb{C}^{m}$ através de funções $F=F(z)$ que são $\bar{\partial}_{z}$-flat sobre 
o traço em $\mathbb{C}^{m}$ da aplicação $Z$, as soluções Gevrey hipo-analíticas também são caracterizadas em $\mathbb{C}^{m} \times \mathbb{R}^{n}$ através de funções $F=F(z, t)$ que são $\left(\bar{\partial}_{z}+\mathrm{d}_{t}\right)$-flat sobre o traço em $\mathbb{C}^{m} \times \mathbb{R}^{n}$ da aplicação $\lambda$. Mais precisamente, a caracterização em $\mathbb{C}^{m} \times \mathbb{R}^{n}$ de $\mathrm{G}_{\mathrm{ha}}^{s}(U)$ se dá através de funções $F=F(z, t)$ infinitamente diferenciáveis no fecho de vizinhanças $\mathcal{W} \subset \mathbb{C}^{m} \times \mathbb{R}^{n}$ de $\lambda(U)$ $\operatorname{com}\left(\bar{\partial}_{z}+\mathrm{d}_{t}\right) F \sim_{\Sigma} 0$, isto é, com $\frac{\partial F}{\partial \bar{z}_{k}}$ e $\frac{\partial F}{\partial \bar{t}_{j}}$ se anulando de ordem infinita em $\Sigma \cap \mathcal{W}$ para todo $k=1, \ldots, m$ e para todo $j=1, \ldots, n$. Dentre elas destacamos as que satisfazem a estimativa (1.14), a saber,

$$
\sup _{\Sigma \cap \overline{\mathcal{W}}}\left|\partial_{z}^{\alpha} F\right| \leq \text { const } \cdot h^{|\alpha|}|\alpha| !^{s}, \forall \alpha \in \mathbb{Z}_{+}^{m} .
$$

Definição 2.4 Vamos denotar por $\mathrm{G}_{\text {ha* }}^{s}(\mathcal{W})$ o espaço das funções $F=F(z, t)$ infinitamente diferenciáveis no fecho de uma vizinhança $\mathcal{W}$ da origem de $\mathbb{C}^{m} \times \mathbb{R}^{n} \operatorname{com}\left(\bar{\partial}_{z}+\mathrm{d}_{t}\right) F \sim_{\Sigma} 0$ e satisfazendo (2.16). O espaço dos germes na origem de tais funçôes será denotado por $\mathrm{G}_{h a \star}^{s}(0)$. Só para evidenciar,

$$
\mathrm{G}_{\text {ha* }}^{s}(\mathcal{W})=\left\{F \in \mathrm{C}^{\infty}(\overline{\mathcal{W}}) \text { com }\left(\bar{\partial}_{z}+\mathrm{d}_{t}\right) F \sim_{\Sigma} 0 \text { e satisfazendo }(2.16)\right\}
$$

Temos então a seguinte caracterização em $\mathbb{C}^{m} \times \mathbb{R}^{n}$ das funções Gevrey hipo-analíticas.

Proposição 2.1 Se $f=f(x, t)$ é infinitamente diferenciável no fecho de uma vizinhança $U \subset \subset \Omega$ da origem e se $s>1$ então são equivalentes:

(i) $f \in \mathrm{G}_{h a}^{s}(U)$;

(ii) existe $F \in \mathrm{G}_{\text {ha* }}^{s}(\mathcal{W}), \mathcal{W} \supset \lambda(U)$, com $\left.F \circ \lambda\right|_{U}=\left.f\right|_{U}$.

\section{Demonstração :}

$\triangleright \quad$ A implicação $(i i) \Rightarrow(i)$ é uma consequência da aplicação da regra da cadeia junto com a dualidade (2.9) e com o anulamento $\left(\bar{\partial}_{z}+\mathrm{d}_{t}\right) F \sim_{\Sigma} 0$; nestas condições vale a igualdade

$$
\begin{gathered}
\left|\mathrm{M}^{\alpha} \mathrm{L}^{\gamma}[F \circ \lambda]\right|=\left|\partial_{z}^{\alpha} \partial_{t}^{\gamma}[F] \circ \lambda\right|, \forall(\alpha, \gamma) \in \mathbb{Z}_{+}^{m+n}, \\
\mathrm{~L}_{j}[F \circ \lambda]=\frac{\partial F}{\partial t_{j}} \circ \lambda=0 \text { para todo } j=1, \ldots, n \text { e }(2.16) \text { implica }(2.10) .
\end{gathered}
$$


Para a implicação $(i) \Rightarrow($ ii $)$ vamos considerar $\Sigma=\lambda(\bar{U})$ como uma subvariedade real maximal de $\mathbb{C}^{m} \times \mathbb{C}^{n} \ni(z, \zeta)$, onde $z=x+i y \in \mathbb{C}^{m}$ e $\zeta=t+i s \in \mathbb{C}^{n}$. A restrição a $s=0$ da solução do problema

$$
\mid \begin{aligned}
& \left(\bar{\partial}_{z}+\bar{\partial}_{\zeta}\right) F \sim_{\Sigma} 0 \\
& \left.F\right|_{\Sigma}=f
\end{aligned}
$$

determina uma função $F(z, t)$ infinitamente diferenciável no fecho de uma vizinhança $\mathcal{W}$ de $\Sigma$ satisfazendo $\left.\bar{\partial}_{z} F \sim_{\Sigma} 0 \operatorname{com} F \circ \lambda\right|_{U}=\left.f\right|_{U}$. A unicidade das soluções para o problema formal de Cauchy

$$
\mid \begin{aligned}
& \left(\bar{\partial}_{z}+\bar{\partial}_{\zeta}\right)\left[\frac{\partial F}{\partial t_{j}}\right] \sim_{\Sigma} 0 \\
& \left.\frac{\partial F}{\partial t_{j}}\right|_{\Sigma}=\mathrm{L}_{j} f=0
\end{aligned}
$$

determina que $\mathrm{d}_{t} F \sim_{\Sigma} 0$.

Vamos trabalhar agora no sentido de mostrar a invariância hipo-analítica

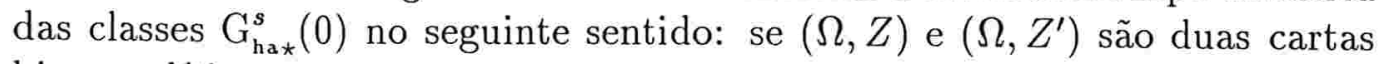
hipo-analíticas com

$$
\begin{array}{ll}
Z=x+i \Phi(x, t) & , \quad \Sigma=\{(Z(x, t), t):(x, t) \in \Omega\}, \\
Z^{\prime}=x^{\prime}+i \Phi^{\prime}\left(x^{\prime}, t^{\prime}\right) & , \quad \Sigma^{\prime}=\left\{\left(Z^{\prime}\left(x^{\prime}, t^{\prime}\right), t^{\prime}\right):\left(x^{\prime}, t^{\prime}\right) \in \Omega\right\}
\end{array}
$$

então a mudança de coordenadas $\left(Z^{\prime}, t^{\prime}\right)=(H(Z), G(Z, t))$ existente induz um isomorfismo natural entre os respectivos espaços $G_{\mathrm{ha} \star}^{s}(0)$ e $\mathrm{G}_{\mathrm{ha} \star^{\prime}}^{s}(0)$ correspondentes.

Proposição $2.2 \mathrm{G}_{h a \star}^{s}(0)$ é um invariante hipo-analítico.

\section{Demonstração :}

$\triangleright \quad$ Iniciamos considerando os espaços $\mathrm{G}_{\mathrm{ha} \text { * }}^{s}(\mathcal{W})$ e $\mathrm{G}_{\mathrm{ha}}^{s}\left(\mathcal{W}^{\prime}\right)$ definidos respectivamente a partir de duas cartas hipo-analíticas $(\Omega, Z)$ e $\left(\Omega, Z^{\prime}\right)$. Vamos considerar também a mudança de coordenadas

$$
\begin{aligned}
Z^{\prime}\left(x^{\prime}, t^{\prime}\right) & =H(Z(x, t)), \quad H=H(z) \text { biholomorfismo } \\
t^{\prime} & =G(Z(x, t), t), \quad G=G(z, t) \text { infinitamente diferenciável }
\end{aligned}
$$


determinada pelo fato da estrutura ser hipo-analítica.

Naturalmente cada função $F^{\prime} \in \mathrm{G}_{\mathrm{ha} \star^{\prime}}^{s}\left(\mathcal{W}^{\prime}\right)$ determina uma função

$$
F(z, t)=F^{\prime}(H(z), G(z, t))=F^{\prime}\left(z^{\prime}, t^{\prime}\right)
$$

infinitamente diferenciável numa vizinhança $\mathcal{W}$ de $\Sigma=H^{-1}\left(\Sigma^{\prime} \cap \mathcal{W}^{\prime}\right)$. Pela regra da cadeia

$$
\begin{aligned}
& \left.\frac{\partial F}{\partial z_{k}}\right|_{\Sigma}=\left.\left.\sum_{k^{\prime}=1}^{m} \frac{\partial F^{\prime}}{\partial z^{\prime} k^{\prime}}\right|_{\Sigma^{\prime}} \frac{\partial H_{k^{\prime}}}{\partial z_{k}}\right|_{\Sigma_{t}}+\left.\left.\sum_{k^{\prime}=1}^{m} \frac{\partial F^{\prime}}{\partial \bar{z}^{\prime}{ }_{k^{\prime}}}\right|_{\Sigma^{\prime}} \frac{\partial \bar{H}_{k^{\prime}}}{\partial z_{k}}\right|_{\Sigma_{t}}+\left.\left.\sum_{j=1}^{n} \frac{\partial F^{\prime}}{\partial t^{\prime} j}\right|_{\Sigma^{\prime}} \frac{\partial G_{j}}{\partial z_{k}}\right|_{\Sigma} \\
& \left.\frac{\partial F}{\partial \bar{z}_{k}}\right|_{\Sigma}=\left.\left.\sum_{k^{\prime}=1}^{m} \frac{\partial F^{\prime}}{\partial z^{\prime} k^{\prime}}\right|_{\Sigma^{\prime}} \frac{\partial H_{k^{\prime}}}{\partial \bar{z}_{k}}\right|_{\Sigma_{t}}+\left.\left.\sum_{k^{\prime}=1}^{m} \frac{\partial F^{\prime}}{\partial \bar{z}^{\prime}{ }_{k^{\prime}}}\right|_{\Sigma^{\prime}} \frac{\partial \bar{H}_{k^{\prime}}}{\partial \bar{z}_{k}}\right|_{\Sigma_{t}}+\left.\left.\sum_{j=1}^{n} \frac{\partial F^{\prime}}{\partial t^{\prime} j}\right|_{\Sigma^{\prime}} \frac{\partial G_{j}}{\partial \bar{z}_{k}}\right|_{\Sigma} \\
& \left.\frac{\partial F}{\partial t_{j}}\right|_{\Sigma}=\left.\left.\sum_{j^{\prime}=1}^{n} \frac{\partial F^{\prime}}{\partial t^{\prime}{ }^{\prime}}\right|_{\Sigma^{\prime}} \frac{\partial G_{j^{\prime}}}{\partial t_{j}}\right|_{\Sigma}
\end{aligned}
$$

segue que

$$
\begin{array}{r}
H \text { holomorfa } \\
\left(\bar{\partial}_{z^{\prime}}+\mathrm{d}_{t^{\prime}}\right) F^{\prime} \sim_{\Sigma^{\prime}} 0
\end{array} \mid \Rightarrow\left(\bar{\partial}_{z}+\mathrm{d}_{t}\right) F \sim_{\Sigma} 0
$$

Por outro lado (cf. [H1, Prop.8.4.1]), as derivações $\partial_{z}^{\alpha} F(Z, t)$ de ordem $\kappa=0,1, \ldots$ coincidem com as mesmas derivações da aplicação

$$
z \mapsto S_{\kappa}(z)=\sum_{|\beta| \leq \kappa} \frac{1}{\beta !} \partial_{z^{\prime}}^{\beta} F^{\prime}(H(Z), G(Z, t))[H(z)-H(Z)]^{\beta}
$$

quando $z=Z$, donde $\left|\partial_{z}^{\alpha} F(Z, t)\right|=\left|\partial_{z}^{\alpha} S_{|\alpha|}(Z)\right|$. Por continuidade vamos considerar um poli-raio $(\rho, \ldots, \rho)$ pequeno a ponto de incluir no domínio de $H$ todos os poli-discos $\Delta(Z, \rho)$ centrados em $Z \in \Sigma_{t}$ e, consequentemente via Fórmula Integral de Cauchy, expressar

$$
\partial_{z}^{\alpha} S_{|\alpha|}(Z)=\frac{\alpha !}{(2 \pi i)^{m}} \int_{\left|z_{k}-Z_{k}\right|=\rho} \frac{S_{|\alpha|}(z)}{(z-Z)^{\alpha+(1, \ldots, 1)}} \mathrm{d} z .
$$

Assim, para todo $(Z, t) \in \mathcal{W} \cap \Sigma$ e para todo $\alpha \in \mathbb{Z}_{+}^{m}$,

$$
\begin{aligned}
\left|\partial_{z}^{\alpha} F(Z, t)\right| & =\left|\partial_{z}^{\alpha} S_{|\alpha|}(Z)\right| \leq \sup _{\left|z_{j}-Z j\right|=\rho}\left|S_{|\alpha|}(z)\right| \cdot \rho^{-|\alpha|} \alpha ! \\
& \leq \sum_{|\beta| \leq|\alpha|} \frac{1}{\beta !} \mid \partial_{z^{\prime}}^{\beta} F^{\prime}\left(H(Z), G(Z, t)\left|\sup _{\left|z_{j}-Z_{j}\right|=\rho}\right|[H(z)-H(Z)]^{\beta} \mid \cdot \rho^{-|\alpha|} \alpha ! .\right.
\end{aligned}
$$


Admitindo que $F^{\prime}$ satisfaz (2.16) em $h_{F^{\prime}}$, que $C_{H}$ é uma constante de Lipshitz satisfazendo $|H(z)-H(Z)| \leq C_{H}|z-Z|$ e utilizando a equivalência $\alpha ! \leq|\alpha| ! \leq m^{|\alpha|} \alpha !$, seque que

$$
\begin{aligned}
\left|\partial_{z}^{\alpha} F(Z, t)\right| & \leq \text { const } \cdot \sum_{|\beta| \leq|\alpha|}\left(h_{F^{\prime}} C_{H} \rho / m\right)^{|\beta|}|\beta| !^{s-1} \cdot \rho^{-|\alpha|}|\alpha| ! \\
& \leq \text { const } \cdot\left(\max \left\{1, h_{F^{\prime}} C_{H} \rho / m\right\}\right)^{|\alpha|}|\alpha| !^{s} \sum_{|\beta| \leq|\alpha|} 1 \\
& \leq \text { const } \cdot\left(2 \max \left\{1, h_{F^{\prime}} C_{H} \rho / m\right\}\right)^{|\alpha|}|\alpha| !^{s}
\end{aligned}
$$

e, portanto, $F$ satisfaz (2.16).

Passemos agora a uma caracterização das classes $\mathrm{G}_{\mathrm{ha}}(0)$ do tipo (1.18).

Definição 2.5 Vamos denotar por $\mathcal{F}_{\Sigma}^{\bullet}(\mathcal{W})$ o espaço das funções $F=F(z, t)$ infinitamente diferenciáveis no fecho de uma vizinhança $\mathcal{W} \subset \subset \mathbb{C}^{m} \times \mathbb{R}^{n} d a$ origem que se anulam de ordem infinita sobre $\Sigma$, isto é,

$$
\mathcal{F}_{\Sigma}^{\bullet}(\mathcal{W})=\left\{F \in C^{\infty}(\overline{\mathcal{W}}) \operatorname{com} F \sim_{\Sigma} 0\right\}
$$

$O$ espaço dos germes na origem de tais funções será denotado por $\mathcal{F}_{\Sigma}^{\bullet}(0)$.

Proposição 2.3 Para todo $s>1$ a sequência

$$
0 \rightarrow \mathcal{F}_{\Sigma}^{\bullet}(0) \longrightarrow \mathrm{G}_{h a \star}^{s}(0) \longrightarrow \mathrm{G}_{h a}^{s}(0) \rightarrow 0
$$

é uma sequência exata curta, garantindo assim o isomorfismo

$$
\mathrm{G}_{h a}^{s}(0) \simeq \mathrm{G}_{h a_{\star}}^{s}(0) / \mathcal{F}_{\dot{\Sigma}}^{*}(0)
$$

\section{Demonstração :}

$\triangleright \quad$ Basta invocarmos a Proposição 2.1 e mostrarmos que o núcleo da aplicação $\mathrm{G}_{\mathrm{ha}}^{s}(0) \rightarrow \mathrm{G}_{\mathrm{ha}}^{s}(0)$ é precisamente $\mathcal{F}_{\Sigma}^{\bullet}(0)$, já que sua sobrejetividade decorre diretamente da proposição invocada. 
Observe que o núcleo em questão contém $\mathcal{F}_{\Sigma}^{\bullet}(0)$, já que o item (ii) da Proposição 2.1 garante que o mesmo é constituído pelas soluções $F=F(z, \zeta)$ infinitamente diferenciáveis do problema formal de Cauchy

$$
\mid \begin{aligned}
& \bar{\partial} F \sim_{\Sigma} 0 \\
& \left.F\right|_{\Sigma}=0
\end{aligned} .
$$

A unicidade das soluções para tal problema garante a inclusão recíproca, isto é, garante que $F \sim_{\Sigma} 0$.

Como $\mathrm{G}_{\mathrm{ha} \star}^{s}(0)$ e $\mathcal{F}_{\Sigma}^{\bullet}(0)$ são invariantes hipo-analíticos, o isomorfismo (2.17) garante a invariância hipo-analítica das classes $\mathrm{G}_{\mathrm{h}}^{s}(0)$. Tal invariância sugere uma generalização do conceito de hipocomplexidade de Treves (cf. [T, III.5]) na seguinte direção:

- uma estrutura hipo-analítica seria dita $s$-hipocomplexa de ordem $s \geq 1$ se toda solução distribuição (ou mesmo hiperfunção) do sistema $L_{j} u=0$ é Gevrey hipo-analítica de ordem $s$.

Quando $m=1$ segue de [T, Cor.III.5.3] que $s$-hipocomplexidade para algum $s$ implica hipocomplexidade. $\mathrm{O}$ estudo para $m \geq 2$ constitui um interessante tema de investigação.

Finalizamos esta seção observando que as funções hipo-analíticas numa vizinhança $U$ da origem introduzidas por Treves (cf.[T, Def.III.1.2]) constituem o espaço das funções Gevrey hipo-analíticas em $U$ de ordem $s=1$ (cf.[T, Prop.III.2.1]) e que quando $s$ percorre $] 1, \infty$ [ obtemos uma modulação entre tais funções e as soluções infinitamente diferenciáveis em $U$.

\subsection{O Teorema de Baouendi-Treves Gevrey hipo-analítico}

Nesta seção vamos demonstrar o teorema de Baouendi-Treves para soluções Gevrey hipo-analíticas enunciado a seguir:

Teorema 2.1 Dada $U \subset \subset \Omega$ vizinhança da origem existe $U^{\prime \prime} \subset \subset U$ vizinhança da origem tal que qualquer solução $f \in \mathrm{G}_{h a}^{s}(U)$ é o limite em $\mathrm{G}_{\mathrm{ML}}^{s}\left(U^{\prime \prime}\right)$ de uma sequência de funções $\left(F_{\tau} \circ Z\right)_{\tau=1,2, \ldots}$ onde cada $F_{\tau}$ é inteira em $\mathbb{C}^{m}$. 
Iniciamos assumindo $U=V \times W \ni(x, t)$ e fixando uma função de corte $\chi \in \mathrm{C}_{c}^{\infty}(V)$ com $\chi$ identicamente um numa vizinhança $V^{\prime} \subset \subset V$ da origem.

Como na Seção 1.5 vamos trabalhar com as fórmulas de aproximação

$$
\tilde{\mathcal{E}}_{\tau}(\chi f)(z, t)=\left(\frac{\tau}{\pi}\right)^{m / 2} \int e^{-\tau\left(z-Z\left(x^{\prime}, t\right)\right\rangle^{2}} \chi\left(x^{\prime}\right) f\left(x^{\prime}, t\right) d Z\left(x^{\prime}, t\right)
$$

e suas respectivas restrições $\mathcal{E}_{\tau}(\chi f)(x, t)=\tilde{\mathcal{E}}_{\tau}(\chi f)(Z(x, t), t)$ a $\Sigma$. Mais precisamente, vamos trabalhar com as funções

$$
\begin{aligned}
H_{\tau}(z, t) & =\tilde{\mathcal{E}}_{\tau}(\chi f)(z, t) \\
G_{\tau}(z, t) & =H_{\tau}(z, t)-H_{\tau}(z, 0)=\int_{0}^{1} \sum_{j=1}^{n} \tilde{\mathcal{E}}_{\tau}\left(L_{j} \chi f\right)(z, \rho t) t_{j} d \rho \\
F_{\tau}(z) & =H_{\tau}(z, 0)=H_{\tau}(z, t)-G_{\tau}(z, t) .
\end{aligned}
$$

Um resultado preliminar com demonstração análoga à demonstração do Teorema 1.2 (pg. 16) é:

- existe $C>0$ tal que, para todo $V^{\prime \prime} \subset \subset V^{\prime}$,

$$
\sup _{V^{\prime \prime} \times W}\left|M^{\alpha}\left[H_{\tau}(Z(x, t), t)\right]\right| \leq C h^{|\alpha|}|\alpha| !^{s}, \forall \alpha \in \mathbb{Z}_{+}^{m}, \forall \tau>0 .
$$

Outro resultado preliminar é obtido através de [T, Lema II.2.4] observando que as funções $\left(L_{j} \chi\right) f$ se anulam identicamente em $V^{\prime} \times W$, a saber,

- existe uma vizinhança $\Delta \times W^{\prime \prime} \subset \subset \mathbb{C}^{m} \times W$ da origem na qual $G_{\tau}$ convergem uniformemente para zero.

Como $H_{\tau} \circ \lambda$ convergem para $\chi f$ uniformemente em compactos de $V \times W$ e como $G_{\tau}$ convergem uniformemente para zero em compactos de $\Delta \times W^{\prime \prime}$, as funções $F_{\tau} \circ Z$ convergem para $\chi f$ em compactos de $U^{\prime \prime} \subset \subset V^{\prime} \times W$ com $\lambda\left(U^{\prime \prime}\right) \subset \Delta \times W^{\prime \prime}$. Basta tomar um tal $U^{\prime \prime}$ e mostrar que a sequência $\left(F_{\tau} \circ Z\right)$ é limitada em algum $G_{\mathrm{ML}}^{s, h}\left(U^{\prime \prime}\right)$, pois assim tal sequência admitirá subsequência convergente (para $f$ ) no espaço de Montel $\mathrm{G}_{\mathrm{ML}}^{s}\left(U^{\prime \prime}\right)$. Mais ainda, basta mostrarmos a limitação em questão somente em relação a $M$, posto que as funções $F_{\tau}$ são inteiras.

Observe que a limitação da sequência $\left(H_{\tau} \circ \lambda\right)$ em $\mathrm{G}_{\mathrm{ML}}^{s, h}\left(U^{\prime \prime}\right)$ para algum $h>0$ já está garantida. 
Com relação à limitação de $\left(G_{\tau} \circ \lambda\right)$, vamos considerar poli-discos $\Gamma_{\rho}(z)$ centrados em $z=Z(x, t) .(x, t) \in \overline{U^{\prime \prime}}$, de poli-raios $(\rho, \ldots, \rho)$ pequenos tal que $\Gamma_{\rho}(z) \subset \Delta$ para todo $(x, t) \in \overline{U^{\prime \prime}}$. Pela Fórmula Integral de Cauchy,

$$
\begin{aligned}
\sup _{\overline{U^{\prime \prime}}}\left|\mathrm{M}^{\alpha}\left[G_{\tau} \circ \lambda\right]\right| & =\sup _{\overline{U^{\prime \prime}}}\left|\partial_{z}^{\alpha}\left[G_{\tau}\right] \circ \lambda\right| \\
& \leq \rho^{-|\alpha|}|\alpha| ! \sup _{(z, t), \tau}\left|G_{\tau}(z, t)\right| \\
& \leq \text { const } \cdot \rho^{-|\alpha|}|\alpha| !, \forall \alpha \in \mathbb{Z}_{+}^{m}, \forall \tau>0
\end{aligned}
$$

donde $(G \circ \lambda)$ é limitada em $\mathrm{G}_{\mathrm{ML}}^{1, \rho^{-1}}\left(U^{\prime \prime}\right)$. 


\section{Capítulo 3}

\section{Resolubilidade Gevrey hipo-analitica parcial}

Este capítulo trata de questões envolvendo a resolubilidade local Gevrey hipo-analítica parcial do complexo de De Rham associado a uma estrutura hipo-analítica de codimensão $n$ sobre uma variedade diferenciável $(m+n)$ dimensional. As análises se dão numa carta hipo-analítica $(\Omega, Z)$ cumprindo as condições (2.1)-(2.9) da Seção 2.1 e o resultado principal estabelece, a partir da resolubilidade $C^{\infty}$, a resolubilidade local do complexo em questão em classes Gevrey parciais segundo a mesma estrutura.

\subsection{O complexo diferencial $\mathbb{L}$}

Nesta seção vamos introduzir o complexo diferencial $\mathbb{L}$ associado à estrutura

$$
\mathrm{T}^{\prime}=\operatorname{Span}\left\{\mathrm{d} Z_{1}, \ldots, \mathrm{d} Z_{m}\right\}
$$

de subfibrado vetorial do fibrado cotangenge complexo $\mathbb{C T}^{*} \mathcal{M}$ sobre $\Omega$. de tipo

Iniciamos pelos espaços das formas diferenciais de grau $q \in\{0,1, \ldots, n\}$

$$
f=\sum_{|J|=q} f_{J}(x, t) \mathrm{d} t_{J}
$$

com coeficientes $f_{J}$ infinitamente diferenciáveis. Aqui estamos assumindo a notação usual de multi-índices, onde $J=\left(j_{1}<j_{2}<\cdots<j_{q}\right)$ e $\mathrm{d} t_{J}$ representa formas $\mathrm{d} t_{j_{1}} \wedge \cdots \wedge \mathrm{d} t_{j_{q}}$ de tamanho $|J|$. 
Como as derivações $L_{1}, \ldots, L_{n}$ definem endomorfismos nos espaços de funções infinitamente diferenciáveis, fica bem definido um complexo $\mathbb{L}$ de operadores diferenciais que leva a $q$-forma $(3.2)$ na $(q+1)$-forma

$$
\mathbb{L} f=\sum_{|J|=q} \sum_{j=1}^{n} \mathrm{~L}_{j} f_{J} \mathrm{~d} t_{j} \wedge \mathrm{d} t_{J} .
$$

Como em [T, Sec.I.6], tal complexo corresponde ao complexo de De Rham em $\left.\mathbb{C T}^{\prime}\right|_{\Omega} \mathcal{M}$ após passagem ao quociente por $\mathrm{T}^{\prime}$.

\subsection{A resolubilidade local $s$-Gevrey hipo-analítica parcial}

Um dos resultados significativos desta tese diz respeito à realização do complexo $\mathbb{L}$ em classes de funções Gevrey parciais segundo $(M, L)$ definidas a partir das estimativas abaixo:

$$
\begin{aligned}
& \text { existe } h>0 \text { tal que para todo } \ell \in \mathbb{Z}_{+} \text {existe } C_{\ell}>0 \text { com } \\
& \sup _{\bar{U}}\left|\mathrm{M}^{\alpha} L^{\gamma} f\right| \leq C_{\ell} h^{|\alpha|}(|\alpha|) !^{s}, \forall(\alpha, \gamma) \in \mathbb{Z}_{+}^{m+n},|\gamma| \leq \ell .
\end{aligned}
$$

Definição 3.1 Dizemos que uma função $f \in C^{\infty}(\bar{U})$ é s-Gevrey parcial segundo $(\mathrm{M}, \mathrm{L})$ em $U$ numa amplitude $h>0$ quando cumpre a estimativa (3.4). O subespaço de $\mathrm{C}^{\infty}(\bar{U})$ constituído por tais funções será denotado por

$$
\mathcal{G}_{\mathrm{ML}}^{s, h}(U)=\left\{f \in \mathrm{C}^{\infty}(\bar{U}): f \text { cumpre }(3.4)\right\}
$$

e a união destes espaços será denotado por

$$
\mathcal{G}_{\mathrm{ML}}^{s}(U)=\bigcup_{h>0} \mathcal{G}_{\mathrm{ML}}^{s, h}(U) .
$$

$O$ espaço dos germes na origem parcialmente Gevrey diferenciáveis segundo $(\mathrm{M}, \mathrm{L})$ será denotado por

$$
\mathcal{G}_{\mathrm{ML}}(0)=\left\{\text { classes } \mathbf{f} \text { representadas por } f \in \mathcal{G}_{\mathrm{ML}}^{s}(U), 0 \in U \subset \subset \Omega\right\} .
$$


É importante ressaltar que (3.4) garante apenas que as derivações com respeito a $L$ numa ordem qualquer $\ell>0$ satisfazem as estimativas Gevrey com respeito a $M$ numa aplitude $h>0$ fixa, independente de $\ell$. Esta independência de $h$ faz com que as classes Gevrey totais definidas a partir das estimativas (2.10) não estejam contidas nas classes Gevrey parciais definidas a partir da estimativa (3.4) (na Seção 5.2 nos comentamos mais a este respeito). Os exemplos a seguir justificam de certa forma a Definição 3.1.

Exemplos de funções em $\mathcal{G}_{\mathrm{ML}}^{1}(U)$ : Considere uma vizinhança da origem em $\mathbb{C}^{m} \times \mathbb{R}^{n}$ do tipo $\Delta \times \Theta$, onde $\Delta$ (resp. $\Theta$ ) é um polidisco aberto (resp. bola aberta) centrado(a) na origem de $\mathbb{C}^{m}\left(\right.$ resp. $\left.\mathbb{R}^{n}\right), \operatorname{com} \lambda(U) \subset \subset \Sigma \cap(\Delta \times \Theta)$. Se $F \in C^{\infty}(\Delta \times \Theta)$ é holomorfa nas variáveis $z_{1}, \ldots, z_{m}$ então existe $h>0$ tal que $F \circ \lambda \in \mathcal{G}_{\text {ML }}^{1, h}(U)$.

A realização do complexo diferencial $\mathbb{L}$ nas classes Gevrey parciais segundo $(M, L)$ passa pelos endomorfismos naturais

$$
\mathrm{L}_{j}: \mathcal{G}_{\mathrm{ML}}^{s}(U) \rightarrow \mathcal{G}_{\mathrm{ML}}^{s}(U), j=1, \ldots, n .
$$

Introduzindo os espaços $\mathcal{G}_{\mathrm{ML}}^{s}\left(U ; \Lambda^{q}\right)$ das $q$-formas diferenciais de tipo (3.2) com coeficientes em $\mathcal{G}_{\text {ML }}^{s}(U)$, os endomorfismos (3.5) definem, via (3.3), um complexo diferencial

$$
\mathbb{L}: \mathcal{G}_{\mathrm{ML}}^{s}\left(U ; \Lambda^{q}\right) \rightarrow \mathcal{G}_{\mathrm{ML}}^{s}\left(U ; \Lambda^{q+1}\right), q=0, \ldots, n-1 .
$$

Com efeito, se $\mathcal{G}_{\mathrm{ML}}^{s}\left(0 ; \Lambda^{q}\right)$ denota germes na origem de formas em $\mathcal{G}_{\mathrm{ML}}^{s}\left(U ; \Lambda^{q}\right)$, temos a sequência

$$
0 \rightarrow \mathcal{G}_{\mathrm{ML}}^{s}\left(0 ; \Lambda^{0}\right) \stackrel{\mathbb{L}}{\rightarrow} \mathcal{G}_{\mathrm{ML}}^{s}\left(0 ; \Lambda^{1}\right) \stackrel{\mathbb{L}}{\rightarrow} \cdots \stackrel{\mathbb{L}}{\rightarrow} \mathcal{G}_{\mathrm{ML}}^{s}\left(0 ; \Lambda^{n}\right) \stackrel{\mathbb{L}}{\rightarrow} 0
$$

cuja cohomologia algébrica associada

$$
\begin{gathered}
H^{0}(0)=\operatorname{Ker}\left[\mathbb{L}: \mathcal{G}_{\mathrm{ML}}^{s}(0) \rightarrow \mathcal{G}_{\mathrm{ML}}^{s}\left(0 ; \Lambda^{1}\right)\right] \\
H^{q}(0)=\frac{\operatorname{Ker}\left[\mathbb{L}: \mathcal{G}_{\mathrm{ML}}^{s}\left(0 ; \Lambda^{q}\right) \rightarrow \mathcal{G}_{\mathrm{ML}}^{s}\left(0 ; \Lambda^{q+1}\right)\right]}{\operatorname{Im}\left[\mathbb{L}: \mathcal{G}_{\mathrm{ML}}^{s}\left(0 ; \Lambda^{q-1}\right) \rightarrow \mathcal{G}_{\mathrm{ML}}^{s}\left(0 ; \Lambda^{q}\right)\right]}, q=1, \ldots, n
\end{gathered}
$$

mede obstruções na resolubilidade local de (3.6) em nível $q$. Observe que $H^{0}(0)=\mathrm{G}_{\mathrm{ha}}^{s}(0)$.

Definição 3.2 Dizemos que o complexo $\mathbb{L}$ é parcialmente s-Gevrey hipoanalítico resolúvel na origem em nível $q \in\{1, \ldots, n\}$ quando $H^{q}(0)=0$ 
ou, equivalentemnte, quando para todo $\mathrm{f} \in \mathcal{G}_{\mathrm{ML}}^{s}\left(0, \Lambda^{q}\right)$ com $\mathbb{L} \mathrm{f}=0$ existe $\mathrm{u} \in \mathcal{G}_{\mathrm{ML}}^{s}\left(0, \Lambda^{q-1}\right)$ com $\mathbb{L u}=\mathrm{f}$. Em termos de representantes, a resolubilidade s-Gevrey hipo-analítica parcial na origem se traduz na seguinte condição:

dada $U \subset \subset \Omega$ vizinhança da origem e dada $f \in \mathcal{G}_{\mathrm{ML}}^{s}\left(U ; \Lambda^{q}\right)$ com $\mathbb{L} f=0$ em $U$ existe $U^{\prime} \subset U$ vizinhança da origem e existe $u \in \mathcal{G}_{\mathrm{ML}}^{s}\left(U^{\prime} ; \Lambda^{q-1}\right)$ com $\mathbb{L} u=f$ em $U^{\prime}$.

A caracterização das classes Gevrey parciais segundo $(M, L)$ em $\mathbb{C}^{m} \times$ $\mathbb{R}^{n}$ utiliza os espaços $\mathcal{G}_{\text {ML }_{\star}}^{s}(0)$ a seguir, que envolvem funções $F=F(z, t)$ infinitamente diferenciáveis no fecho de vizinhanças $\mathcal{W}$ da origem de $\mathbb{C}^{m} \times \mathbb{R}^{n}$ cumprindo a estimativa (3.4) complexificada, a saber,

$$
\begin{aligned}
& \text { existe } h>0 \text { tal que para todo } \ell \in \mathbb{Z}_{+} \text {existe } C_{\ell}>0 \text { com } \\
& \sup _{\overline{\mathcal{W}}}\left|\partial_{z}^{\alpha} \partial_{t}^{\gamma} F\right| \leq C_{\ell} h^{|\alpha|}|\alpha| !^{s}, \forall(\alpha, \gamma) \in \mathbb{Z}_{+}^{m+n},|\gamma| \leq \ell
\end{aligned}
$$

Definição 3.3 Vamos denotar por $\mathcal{G}_{\mathrm{ML}_{*}}^{s}(\mathcal{W})$ o espaço das funções $F=F(z, t)$ infinitamente diferenciáveis no fecho de uma vizinhnaça $\mathcal{W}$ da origem de $\mathbb{C}^{m} \times \mathbb{R}^{n}$ com $\bar{\partial}_{z} F \sim_{\Sigma} 0$ e satisfazendo (3.10). Só para evidenciar

$$
\mathcal{G}_{\mathrm{ML}{ }_{*}}^{s}(\mathcal{W})=\left\{\mathrm{F}: F \in \mathrm{C}^{\infty}(\overline{\mathcal{W}}) \text { com } \bar{\partial}_{z} F \sim_{\Sigma} 0 \text { e satisfazendo }(3.10)\right\} \text {. }
$$

$O$ espaço dos germes na origem de tais funções será denotado por $\mathcal{G}_{\mathrm{ML}_{\star}}^{s}(0)$.

Proposição 3.1 Se $f=f(x, t)$ é infinitamente diferenciável no fecho de uma vizinhança $U \subset \subset \Omega$ da origem e se $s>1$ então são equivalentes:

(i) $f \in \mathcal{G}_{\text {ML }}^{s}(U)$;

(ii) existe $F \in \mathcal{G}_{\mathrm{ML}_{\star}}^{s}(\mathcal{W}), \mathcal{W} \supset \lambda(U)$, com $F \circ \lambda=f$.

\section{Demonstração :}

$\triangleright \quad$ A implicação $(i i) \Rightarrow(i)$ é uma consequência imediata da aplicação da regra da cadeia junto com a dualidade (2.9) e o anulamento $\bar{\partial}_{z} F \sim_{\Sigma} 0$, já que neste caso $\mathrm{M}^{\alpha} \mathrm{L}^{\gamma}[F \circ \lambda]=\partial_{z}^{\alpha} \partial_{t}^{\gamma}[F] \circ \lambda$ e (3.10) implica (3.4).

Para a implicação $(i) \Rightarrow($ ii $)$ vamos considerar, como na Proposição 2.1, uma função $F=F(z, t)$ infinitamente diferenciável numa vizinhança $\lambda(U) \subset \mathcal{W} \supset \mathbb{C}^{m} \times \mathbb{R}^{n}$ satisfazendo $\bar{\partial}_{z} F \sim_{\Sigma} 0$ com $\left.F\right|_{\Sigma}=f$. Tal função, via $\mathrm{M}^{\alpha} \mathrm{L}^{\gamma}[F \circ \lambda]=\partial_{z}^{\alpha} \partial_{t}^{\gamma}[F] \circ \lambda$ e estimativa (3.4), representa um elemento em $\mathcal{G}_{\mathrm{ML}_{*}}^{s}(\mathcal{W})$. 


\subsection{Resolubilidade $C^{\infty}$ versus resolubilidade Gevrey hipo-analítica}

Nesta seção estabelecemos a resolubilidade local $s$-Gevrey hipo-analítica parcial do complexo $\mathbb{L}$ para todo $s>1$ a partir da sua resolubilidade local clássica $C^{\infty}$, caracterizada em nível $q$ pela condição

dada $U \subset \subset \Omega$ vizinhança da origem existe $U^{\prime} \subset U$ vizinhança da origem tal que para toda $f \in \mathrm{C}^{\infty}\left(U ; \Lambda^{q}\right)$ com $\mathbb{L} f=0 \mathrm{em}$ $U$ existe $u \in \mathcal{C}^{\infty}\left(U^{\prime} ; \Lambda^{q-1}\right)$ com $\mathbb{L} u=f$ em $U^{\prime}$.

Aqui $\mathrm{C}^{\infty}\left(U ; \Lambda^{q}\right)$ denota o espaço das formas diferenciais de grau $q$ de tipo (3.2) com coeficientes em $\mathrm{C}^{\infty}(\bar{U})$.

Teorema 3.1

$$
(3.11) \Rightarrow(3.9), \forall s>1, \forall q=1, \ldots, n .
$$

A demonstração do teorema acima utiliza adaptações dos Lemas 3, 4 e 5 da Seção 1.5 para as classes Gevrey parciais segundo (M, L). Lembramos que tais lemas envolvem operadores Gevrey diferenciais do tipo

$$
P_{r}(\mathrm{M})=\prod_{p=1}^{\infty}\left(1-\frac{r^{2}}{p^{2 s}}\left[\mathrm{M}_{1}^{2}+\cdots+\mathrm{M}_{m}^{2}\right]\right), r>0 .
$$

A adaptação dos Lemas 3 e 4 utiliza as estimativas (1.36) com parâmetros, em vizinhanças complexas de fatias $\Sigma_{t}$ do tipo

$$
\Sigma_{t, \delta}=\left\{z+i v: z \in \Sigma_{t}, v \in \mathbb{R}^{m},|v|<\delta\right\}
$$

e em n-uplas complexas $\theta_{t}$ expressas na forma

$$
\theta_{t}=\zeta+i(z-w)\langle\zeta\rangle, z \in \Sigma_{t}, \zeta \in \mathbb{R T}_{z}^{\prime} \Sigma_{t}, w \in \mathbb{C}^{m} .
$$

Trabalhando como em [C, Sec.2] numa pequena vizinhança $U \subset \subset \Omega$ da origem e com números reais $\kappa, \delta_{0}>0$ também pequenos, é possível validar (1.36) com $\theta_{t}$ no lugar de $\theta, z=Z(x, t), z^{\prime}=Z\left(x^{\prime}, t\right), \zeta \in \mathbb{R T}_{z^{\prime}}^{\prime} \Sigma_{t}, v \in \mathbb{R}^{m}$ com $|v| \leq 1$ e $w \in \Sigma_{t, \delta_{0}}$ para todo $(x, t) \in U$. Neste contexto os lemas em questão são facilmente adaptados através dos lemas a seguir. 
Lema 6 Fixado $r \in] 0,1\left[\right.$ existe $h_{r}>0$ tal que qualquer função $v \in C^{\infty}(U)$ é representada numa vizinhança $U^{\prime} \subset \subset U$ da origem por $P_{r}(\mathrm{M}) w=v$ onde $w \in \mathcal{G}_{\mathrm{ML}}^{s, h_{r}}\left(U^{\prime}\right)$.

\section{Demonstração :}

$\triangleright \quad$ Basta repetir a demonstração do Lema 3 com funções $v_{\epsilon}=v_{\epsilon}(x, t)$ definidas através das representações integrais

$$
\frac{1}{(2 \pi)^{m}} \int_{x^{\prime} \in \mathbb{R}^{m}} \int_{\xi \in \mathbb{R}^{m}} \frac{e^{i \xi \cdot\left(Z(x, t)-Z\left(x^{\prime}, t\right)-\epsilon\langle\xi)^{2}\right.}}{P_{r}(\xi)} \chi\left(x^{\prime}\right) v\left(x^{\prime}, t\right) \operatorname{det} Z_{x}\left(x^{\prime}, t\right) \mathrm{d} \xi \mathrm{d} x^{\prime} .
$$

A única diferença é a expressão das derivações $M^{\alpha} L^{\gamma} v_{\epsilon}$ de ordem $|\gamma|<\ell$, a saber,

$$
\frac{1}{(2 \pi)^{m}} \int_{\Sigma_{t}} \int_{\mathbb{R} T_{Z^{\prime}}^{\prime} \Sigma_{t}} \frac{(i \theta)^{\alpha} e^{i \theta \cdot\left(Z-Z^{\prime}\right)-\epsilon\langle\theta\rangle^{2}}}{P_{r}(\theta) \prod\left(1+\left|\theta_{k}\right|^{2}\right)} \prod\left(1-\mathrm{M}_{k}^{2}\right) L^{\gamma}[\chi v] \Delta\left(Z-Z^{\prime}, \zeta\right) \mathrm{d} \zeta \mathrm{d} Z^{\prime}
$$

que torna a constante em

$$
\text { const } \cdot\left(\sqrt{\frac{11}{13}} r\right)^{-|\alpha|}|\alpha| !^{s}\left(1+|\theta|^{2}\right)^{-m}
$$

dependente de $\ell$.

O próximo lema é uma aplicação direta do Teorema $2.2 \mathrm{em} \mathrm{[C].}$

Lema 7 Fixada qualquer vizinhança $U^{\prime} \subset \subset U$ da origem sempre existe uma vizinhança $\mathcal{W} \subset \mathbb{C}^{m} \times \mathbb{R}^{n}$ de $\lambda\left(U^{\prime}\right)$ na qual as soluçôes $g \in \mathcal{G}_{\mathrm{ML}}^{\text {s }}(U)$ de $P_{r}(\mathrm{M}) g=0$ se estendem holomorficamente em $z$, isto é, existe $G \in \mathrm{C}^{\infty}(\mathcal{W})$ holomorfa em $z$ com $\left.G \circ \lambda\right|_{U^{\prime}}=\left.g\right|_{U^{\prime}}$.

Com relação ao Lema 5 , temos

Lema 8 Fixado $h>0$ existe $r_{h}>0$ tal que, para todo $s>1$ e para toda vizinhança $U \subset \subset \Omega$ da origem, os operadores $P_{r}(\mathrm{M})$ com $0<r \leq r_{h}$ definem aplicações

$$
P_{r}(\mathrm{M}): \mathcal{G}_{\mathrm{ML}}^{s, h}(U) \rightarrow \mathrm{C}^{\infty}(U)
$$




\section{Demonstração :}

$\triangleright \quad$ A única diferença na demonstração do Lema 5 é que as derivações $\mathrm{M}^{\alpha} \mathrm{L}^{\gamma}\left[a_{\alpha^{\prime}} \mathrm{M}^{\alpha^{\prime}} f\right]$ de ordem $|\gamma| \leq \ell$ vão satisfazer

$$
\sup _{\bar{U}}\left|\mathrm{M}^{\alpha} L^{\gamma}\left[a_{\alpha^{\prime}} \mathrm{M}^{\alpha^{\prime}} f\right]\right| \leq B_{2} C_{\ell} C_{\epsilon}\left((1+\epsilon) H_{2} \sqrt{m} H h r\right)^{\left|\alpha^{\prime}\right|}((1+\epsilon) h)^{|\alpha|+\ell}(|\alpha|+\ell) !^{s} .
$$

\section{Demonstração do Teorema 3.1 :}

De início vamos trabalhar numa pequena vizinhança $\Omega$ da origem e com números $\kappa$ e $\delta_{0}$ pequenos para podermos utilizar os lemas demonstrados até agora.

Fixemos $U \subset \subset \Omega$ vizinhança da origem e $f \in \mathcal{G}_{\mathrm{ML}}^{s, h}\left(U ; \Lambda^{q}\right)$ com $\mathbb{L} f=0$ em $U$.

Vamos considerar, via Lema 8, um operador $P(\mathrm{M})$ do tipo (3.12) tal que

$$
P(M): \mathcal{G}_{\mathrm{ML}}^{s, h}\left(U ; \Lambda^{q}\right) \rightarrow \mathrm{C}^{\infty}\left(U ; \Lambda^{q}\right),
$$

onde a atuação de $P(\mathrm{M})$ se dá nos coeficientes das formas.

A comutatividade entre $\mathrm{M}_{k}$ e $\mathrm{L}_{j}$ e a constância dos coeficientes complexos de $P(\mathrm{M})$ faz com que $\mathbb{L} P(\mathrm{M})=P(\mathrm{M}) \mathbb{L}$, donde

$$
\mathbb{L}[P(\mathrm{M}) f]=P(\mathrm{M})[\mathbb{L} f]=0 \text { em } U .
$$

Através da resolubilidade clássica (3.11), existe uma vizinhança $U_{1} \subset U$ da origem (universal em relação a $f$ e $P(\mathrm{M})$ ) e existe uma $(q-1)$-forma

$$
v \in \mathrm{C}^{\infty}\left(\bar{U}_{1} ; \Lambda^{q-1}\right) \quad \text { com } \quad \mathbb{L} v=P(\mathrm{M}) f \text { em } U_{1} .
$$

Pelo Lema 6, existe uma vizinhança $U_{2} \subset \subset U_{1}$ da origem e existe uma $(q-1)$-forma

$$
w \in \mathcal{G}_{\mathrm{ML}}^{s}\left(U_{2} ; \Lambda^{q-1}\right) \quad \text { com } \quad P(\mathrm{M}) w=v \text { em } U_{2} .
$$


Podemos então definir

$$
g=\mathbb{L} w-\left.f\right|_{\overline{U_{2}}} \quad \text { com } \quad P(\mathrm{M}) g=0 \text { em } \quad U_{2},
$$

já que $P(\mathrm{M}) g=P(\mathrm{M})[\mathbb{L} w]-P(\mathrm{M}) f=\mathbb{L}[P(\mathrm{M}) w]-P(\mathrm{M}) f=\mathbb{L} v-P(\mathrm{M}) f$.

Pelo Lema 7, existe uma vizinhança $U_{3} \subset U_{2}$ da origem, existe uma vizinhança $\mathcal{U}_{3}$ de $\lambda\left(U_{3}\right)$ em $\mathbb{C}^{m} \times \mathbb{R}^{n}$ e existe uma $q$-forma

$$
G \in \mathrm{C}^{\infty}\left(\mathcal{U}_{3} ; \Lambda^{q}\right) \text { [holomorfa em } z \text { ] com }\left.G \circ \lambda\right|_{U_{3}}=\left.g\right|_{U_{3}} .
$$

A $q$-forma $G$ é $d_{t}$-fechada em $\lambda\left(U_{3}\right)$, já que

$$
\left.d_{t} G\right|_{\lambda\left(U_{3}\right)}=\left.\mathbb{L}[G \circ \lambda]\right|_{U_{3}}=\mathbb{L}(\mathbb{L} w-f)=0 .
$$

O Lema de Poincaré garante a existência de uma vizinhança $U^{\prime} \subset U_{2}$ da origem, uma vizinhança $\mathcal{U}^{\prime} \subset \mathcal{U}_{3}$ de $\lambda\left(U^{\prime}\right)$ em $\mathbb{C}^{m} \times \mathbb{R}^{n}$ e uma $(q-1)$-forma

$$
H \in C^{\infty}\left(\mathcal{U}^{\prime} ; \Lambda^{q-1}\right) \text { [holomorfa em } z \text { ] com }\left.d_{t} H\right|_{\lambda\left(U^{\prime}\right)}=\left.G\right|_{\lambda\left(U^{\prime}\right)} .
$$

Basta definir então

$$
u=\left.w\right|_{\overline{U^{\prime}}}-\left.H \circ \lambda\right|_{\overline{U^{\prime}}}
$$

e observar que, em $U^{\prime}$,

$$
\mathbb{L} u=\mathbb{L} w-\mathbb{L}[H \circ \lambda]=\mathbb{L} w-d_{t} H \circ \lambda=g+f-G \circ \lambda=f .
$$

$\mathrm{O}$ fato dos coeficientes de $H$ serem infinitamente diferenciável em $\mathcal{U}^{\prime}$ e holomorfos nas variáveis $z_{1}, \ldots, z_{m}$ os inclui, como nos exemplos da pg.40, em $\mathcal{G}_{\mathrm{ML}}^{1, h^{\prime}}$ para algum $h^{\prime}$ dependendo somente da expessura de $\mathcal{U}^{\prime}$ em relação à $\lambda\left(U^{\prime}\right)$, isto é, dependendo somente de $\delta_{0}$ (cf.[C]). Assim

$$
H \circ \lambda \in \mathcal{G}_{\mathrm{ML}}^{1}\left(U^{\prime} ; \Lambda^{q-1}\right) \subset \mathcal{G}_{\mathrm{ML}}^{s}\left(U^{\prime} ; \Lambda^{q-1}\right)
$$

donde

$$
u \in \mathcal{G}_{\mathrm{ML}}^{s}\left(U^{\prime} ; \Lambda^{q-1}\right)
$$




\section{Capítulo 4}

\section{Resolubilidade Gevrey para funções $(q=1)$}

Este capítulo trata de questões envolvendo a resolubilidade local Gevrey hipo-analítica do complexo $\mathbb{L}$ em $q=1$. As análises se dão como no capítulo anterior e o resultado principal utiliza a mesma técnica da demonstração do Teorema 3.1 para estabelecer a resolubilidade local de $\mathbb{L}$ nas classes Gevrey (totais) segundo $(\mathrm{M}, \mathrm{L})$ a partir da resolubilidade clássica $\mathrm{C}^{\infty}$. Uma consequência imediata é a suficiência da condição $(\mathcal{P})$ de Niremberg-Treves para a resolubilidade Gevrey local de operadores diferenciais parciais lineares de primeira ordem com coeficientes analíticos não degenerados na origem.

\subsection{Resolubilidade local em funções Gevrey segundo $(M, L)$}

Nesta seção vamos apresentar algumas questões relevantes envolvendo a resolubilidade local do complexo diferencial $\mathbb{L}$ em classes de funções Gevrey diferenciáveis segundo (M, L), definidas na Seção 2.2 .

A realização de $\mathbb{L}$ em tais classes utiliza a seguinte proposição.

Proposição 4.1 As derivações $\mathrm{L}_{1} \ldots, \mathrm{L}_{n}$ definem aplicações

$$
\mathrm{L}_{j}: \mathrm{G}_{\mathrm{ML}}^{s, h}(U) \rightarrow \mathrm{G}_{\mathrm{ML}}^{s, h^{+}}(U), h^{+}>h
$$

em qualquer vizinhança $U \subset \subset \Omega$ da origem e em qualquer $s \geq 1$. 


\section{Demonstração :}

$\triangleright$ A proposição segue da estabilidade sobre operadores diferenciais da sequência $\left\{p !^{s}\right\}$, que se traduz na estimativa

$$
\forall \epsilon>0 \exists C_{\epsilon}:(p+1) !^{s} \leq C_{\epsilon}(1+\epsilon)^{p} p !^{s}, \forall p \geq 0 .
$$

Fixada $f \in \mathrm{G}_{\mathrm{ML}}^{s, h}(U)$, tal estabilidade permite estimar

$$
\begin{aligned}
\sup _{\bar{U}}\left|\mathrm{M}^{\alpha} \mathrm{L}^{\beta}\left[\mathrm{L}_{j} f\right]\right| & \leq\|f\|_{s, h, U} h^{|\alpha|+|\beta|+1}(|\alpha|+|\beta|+1) !^{s} \\
& \leq\|f\|_{s, h, U} C_{\epsilon} h \cdot((1+\epsilon) h)^{|\alpha|+|\beta|} \cdot(|\alpha|+|\beta|) !^{s} .
\end{aligned}
$$

De acordo com a proposição anterior. o complexo $\mathbb{L}$ se realiza nas classes de funções Gevrey segundo (M, L), via (3.3), definindo aplicações

$$
\begin{aligned}
& \mathbb{L}: \mathrm{G}_{\mathrm{ML}}^{s, h}(U) \rightarrow \mathrm{G}_{\mathrm{ML}}^{s, h^{+}}\left(U ; \Lambda^{1}\right), \forall h<h_{+} \\
& \mathbb{L}: \mathrm{G}_{\mathrm{ML}}^{s}(U) \rightarrow \mathrm{G}_{\mathrm{ML}}^{s}\left(U: \Lambda^{1}\right), \\
& \mathbb{L}: \mathrm{G}_{\mathrm{ML}}^{s}(0) \rightarrow \mathrm{G}_{\mathrm{ML}}^{s}\left(0 ; \Lambda^{1}\right),
\end{aligned}
$$

e a resolubilidade Gevrey hipo-analítica local em $q=1$ se traduz em termos de germes na origem na condição

dada $\mathrm{f}_{1}, \ldots, \mathrm{f}_{n} \in \mathrm{G}_{\mathrm{ML}}^{s}(0)$ com $\mathrm{L}_{j} \mathrm{f}_{j^{\prime}}=\mathrm{L}_{j^{\prime}} \mathrm{f}_{j}$ para todo $j, j^{\prime} \in\{1, \ldots, n\}$ existe $\mathbf{u} \in \mathrm{G}_{\mathrm{ML}}^{s}(0)$ com $\mathrm{L}_{j} \mathbf{u}=\mathrm{f}_{j}$ para todo $j \in\{1, \ldots, n\}$.

ou, em termos de seus representantes, na condição

dados $U \subset \subset \Omega$ vizinhança da origem, $h>0$ e $f_{1}, \ldots, f_{n} \in \mathrm{G}_{\mathrm{ML}}^{s, h}(U)$ com $L_{j} f_{j^{\prime}}=L_{j^{\prime}} f_{j}$ em $U$ para todo $j, j^{\prime} \in\{1, \ldots, n\}$, existem $U^{\prime} \subset U$ vizinhança da origem, $h^{\prime}>0$ e $u \in \mathrm{G}_{\mathrm{ML}}^{s, h^{\prime}}\left(U^{\prime}\right)$ com $\mathrm{L}_{j} u=f_{j}$ em $U^{\prime}$ para todo $j \in\{1, \ldots, n\}$.

Como na resolubilidade local $\mathrm{C}^{\infty}$, uma aplicação do Teorema de Baire torna (4.6) equivalente à formulação a seguir, onde $U^{\prime}$ e $h^{\prime}$ são tomadas independentes de $f_{1}, \ldots, f_{n}$, a saber,

dados $U \subset \subset \Omega$ vizinhança da origem e $h>0$ existem $U^{\prime} \subset U$ vizinhança da origem e $h^{\prime}>0$ tais que, se $f_{1}, \ldots, f_{n} \in \mathrm{G}_{\mathrm{ML}}^{s, h}(U)$ satisfaz $L_{j} f_{j^{\prime}}=L_{j^{\prime}} f_{j}$ em $U$ para todo $j, j^{\prime} \in\{1, \ldots, n\}$ então existe $u \in \mathrm{G}_{\mathrm{ML}}^{s, h^{\prime}}\left(U^{\prime}\right)$ satisfazendo $\mathrm{L}_{j} u=f_{j}$ em $U^{\prime}$ para todo $j \in\{1, \ldots, n\}$. 
A aplicação consiste em considerar espaços

$$
\begin{aligned}
E & =\left\{f=\left(f_{1}, \ldots, f_{n}\right): \mathbb{L} f=0\right\} \subset \mathrm{G}_{\mathrm{ML}}^{s, h}\left(U ; \Lambda^{1}\right) \\
E_{\ell} & =\{(u, f): \mathbb{L} u=f\} \subset \mathrm{G}_{\mathrm{ML}}^{s, \ell}\left(U_{\ell}\right) \times E
\end{aligned}
$$

onde $U_{\ell}$ são vizinhanças se exaurindo a origem; a formulação (4.6) garante que $E$ é a união das projeções $\pi_{\ell}(u, f)=f$; pelo teorema de Baire, existem $U^{\prime}=U_{\ell_{0}}$ e $h^{\prime}=\ell_{0}$ tais que $\pi_{\ell_{0}}\left(E_{\ell_{0}}\right)$ não é magro; pelo teorema da aplicação aberta, $\pi_{\ell_{0}}: E_{\ell_{0}} \rightarrow E$ é sobrejetora.

Uma variante forte de (4.7) ocorre quando $U^{\prime}$ independe de $h$, a saber,

dados $U \subset \subset \Omega$ vizinhança da origem existe $U^{\prime} \subset U$ vizinhança da origem tal que, se $f_{1}, \ldots, f_{n} \in \mathrm{G}_{M L}^{s}(U)$ satisfaz $\mathrm{L}_{j} f_{j^{\prime}}=\mathrm{L}_{j^{\prime}} f_{j}$ em $U$ para todo $j, j^{\prime} \in\{1, \ldots, n\}$ então existe $u \in \mathrm{G}_{\mathrm{ML}}^{s}\left(U^{\prime}\right)$ satisfazendo $\mathrm{L}_{j} u=f_{j}$ em $U^{\prime}$ para todo $j \in\{1, \ldots, n\}$.

Um exemplo interessante onde vale (4.7) e não vale (4.8) é a estrutura de Mizohata no plano $\mathbb{R}_{x} \times \mathbb{R}_{t}$, definida pela carta $Z=x+i t^{2} / 2$. Neste exemplo a diferenciabilidade Gevrey usual e a diferenciabilidade Gevrey segundo a estrutura de Mizohata coincidem, posto que $Z$ é analítica. O complexo $\mathbb{L}$ se reduz ao único campo $L=\frac{\partial}{\partial t}-i t \frac{\partial}{\partial x}$. As funções em $\mathrm{G}^{1, h}(U)$ correspondem às analíticas reais em $\bar{U}$ que se estendem holomorficamente numa vizinhança $\mathcal{U}=\left\{z \in \mathbb{C}^{2}: \operatorname{dist}(z, \bar{U})<h^{-1}\right\}$ de $\bar{U}$. A condição (4.7) é uma consequência imediata do Teorema de Cauchy. Por outro lado, o teorema [H, Teo.6.1.4] garante que:

existe uma vizinhança $U$ da origem tal que, para toda $U^{\prime} \subset \subset U$, existe uma função $f$ tornando o problema $L u=f$ sem solução em $\mathcal{D}^{\prime}\left(U^{\prime}\right)$, embora $f$ possa ser estendida holomorficamente numa vizinhança complexa $\mathcal{U}$ de $U$.

Mais precisamente, dada $U^{\prime} \subset \subset U$ existe uma amplitude $h>0$ e existe uma função $f \in \mathrm{G}^{1, h}(U)$ tal que a equação $\mathrm{L} u=f$ não possui solução em $\mathcal{D}^{\prime}\left(U^{\prime}\right)$ para todo $U^{\prime} \subset U$; em particular, a equação $L u=f$ não possui solução em $\mathrm{G}^{1}\left(U^{\prime}\right)$ para todo $U^{\prime} \subset U$, contrariando (4.8).

Passemos agora a um fato importante sobre a resolubilidade Gevrey hipoanalítica que ocorre no nível $q=1$. 
Proposição 4.2 Se $f \in \mathrm{G}_{\mathrm{ML}}^{s, h}\left(U ; \Lambda^{1}\right)$ é tal que $\mathbb{L} f=0$ em $U$ e se $u \in \mathrm{C}^{\infty}\left(U^{\prime}\right)$ é tal que $\mathbb{L} u=f$ em $U^{\prime}$ então

$$
\sup _{\overline{U^{\prime}}}\left|\mathrm{M}^{\alpha} \mathrm{L}^{\gamma} u\right| \leq \mathrm{const} \cdot h^{|\alpha|+|\gamma|}(|\alpha|+|\gamma|) !^{s}, \forall(\alpha, \gamma) \in \mathbb{Z}_{+}^{m+n},|\gamma|>0 .
$$

\section{Demonstração :}

$\triangleright \quad$ As estimativas em questão decorrem diretamente das estimativas (2.10) para os coeficientes $f_{1}, \ldots, f_{n}$ de $f$. Por exemplo, supondo que $\gamma_{1}>0$ temos

$$
\begin{aligned}
\sup _{\bar{U}^{\prime}}\left|\mathrm{M}^{\alpha} \mathrm{L}^{\gamma} u\right| & =\sup _{\bar{U}^{\prime}}\left|\mathrm{M}^{\alpha} \mathrm{L}^{\left(\gamma_{1}-1, \gamma_{2}, \ldots, \gamma_{n}\right)} \mathrm{L}_{1} u\right| \\
& =\sup _{\bar{U}^{\prime}}\left|\mathrm{M}^{\alpha} \mathrm{L}^{\left(\gamma_{1}-1, \gamma_{2}, \ldots, \gamma_{n}\right)} f_{1}\right| \\
& \leq \| f_{1}||_{s, h, U} h^{|\alpha|+|\gamma|-1}(|\alpha|+|\gamma|-1) !^{s} \\
& \leq \frac{\| f_{1}||_{s, h, U}}{h} h^{|\alpha|+|\gamma|}(|\alpha|+|\gamma|) !^{s} .
\end{aligned}
$$

$\triangleleft$

A Proposição 4.2 e a técnica da demonstração do Teorema 3.1 viabilizam o seguinte teorema.

Teorema 4.1 Se $s>1$ e se $q=1$ então

$$
(3.11) \Rightarrow(4.8) \text {. }
$$

\section{Demonstração :}

Basta observar que os lemas da Seção 3.3 também se aplicam em $\mathrm{G}_{\mathrm{ML}}^{s, h}(U)$. Com isto, podemos reproduzir a demonstração do Teorema 3.1 e obter uma solução $u \in C^{\infty}\left(\overline{U^{\prime}}\right)$ de $\mathbb{L} u=f$ satisfazendo as estimativas (2.10) para todo $(\alpha, \gamma) \in \mathbb{Z}_{+}^{m+n}$ com $|\gamma|=0$.

A Proposição 4.2 garante tais estimativas para $|\gamma|>0$.

Concluímos então que $u \in \mathrm{G}_{\mathrm{ML}}^{s}\left(U^{\prime}\right)$. 


\subsection{A condição $(\mathcal{P})$ versus resolubilidade Gevrey}

Nesta seção vamos tratar da resolubilidade Gevrey de operadores diferenciais parciais lineares de primeira ordem não degenerados na origem de $\mathbb{R}^{m+1}, m \geq 1$.

De acordo com [T2], podemos considerar um sistema de coordenadas

$$
\Omega=B \times \Theta \ni(x, t)=\left(x_{1}, \ldots, x_{m}, t\right)
$$

centrado na origem onde a parte principal dos operadores em questão (sem o termo de ordem zero) se reduz, módulo multiplicações por funções não nulas em $\Omega$, à forma standard

$$
\mathrm{L}=\frac{\partial}{\partial t}+i \sum_{k=1}^{m} b_{k}(x, t) \frac{\partial}{\partial x_{k}},
$$

onde $b=\left(b_{1}, \ldots, b_{m}\right)$ é uma função infinitamente diferenciável em $\Omega$ a valores em $\mathbb{R}^{m}$.

A condição $(\mathcal{P})$ de Nirenberg-Treves, a saber,

$$
\begin{aligned}
& \text { para todo } \xi \in \mathbb{R}^{m} \text { e para todo } x \in B \text {, a função que a } \\
& \text { cada } t \in \Theta \text { associa } b(x, t) \cdot \xi \in \mathbb{R} \text { não muda de sinal. }
\end{aligned}
$$

garante a existência de $m$ soluções $Z_{1}, \ldots, Z_{m}$ para o problema $L u=0$, soluções estas infinitamente diferenciáveis com $\mathrm{d} Z_{1}, \ldots, \mathrm{d} Z_{m}$ linearmente independentes em $\Omega$. Com efeito, podemos considerar as classes $\mathrm{G}_{\mathrm{ML}}^{s}(U)$ de funções Gevrey diferenciáveis segundo a base $(M, L)=\left(M_{1}, \ldots, M_{m}, L\right)$ dual de $\left(\mathrm{d} Z_{1}, \ldots, \mathrm{d} Z_{m}, \mathrm{~d} t\right)$. Mais ainda, a condição $(\mathcal{P})$ também é suficiente para a resolubilidade local $\mathrm{C}^{\infty}$ de $\mathrm{L}$.

Segue então o seguinte corolário do Teorema 4.1:

Corolário 4.1.1 Todo operador diferencial parcial linear $L$ satisfazendo a condição $(\mathcal{P})$ de Nirenberg-Treves é localmente Gevrey resolúvel nas classes de Gevrey de ordem s $>1$ definidas a partir da estrutura localmente integrável gerada por $\mathrm{L}$.

Em particular, segue da Proposição 1.3 um dos principais resultados deste trabalho: 
Teorema 4.2 A condição $(\mathcal{P})$ de Nirenberg-Treves é suficiente para a resolubilidade Gevrey local de operadores linares de primeira ordem não degenerados com coeficientes analíticos. 


\section{Capítulo 5}

\section{Observações Finais}

\subsection{A questão da existência de funções cortes nas classes de Gevrey segundo $M$}

A questão da existência de funções cortes em $G_{M}^{s}(V)$ no caso em que $Z_{1}, \ldots, Z_{m}$ não são usualmente $s$-Gevrey não foi respondida nesta tese. Utilizando a teoria de feixes desenvolvida em $[\mathrm{Br}]$ vamos dar indícios da não existência de tais cortes neste caso particular em que a subvariedade $\Sigma$ não é $s$-Gevrey. Por comodidade vamos assumir $m=1$.

Iniciamos listando os feixes abaixo construídos a partir dos respectivos pré-feixes:

$$
\begin{aligned}
\mathrm{G}_{\mathrm{M}}^{s} & : B \supset V \mapsto \mathrm{G}_{\mathrm{M}}^{s}(V) \\
\mathrm{G}^{s} & : B \supset V \mapsto \mathrm{G}^{s}(V) \\
\mathcal{G}^{s} & : \mathbb{C} \supset \mathcal{U} \mapsto \mathrm{G}^{s}(\mathcal{U}) \\
\mathcal{G}_{\star}^{s} & : \mathbb{C} \supset \mathcal{U} \mapsto \mathcal{G}_{\star}^{s}(\mathcal{U})=\left\{F \in \mathrm{G}^{s}(\mathcal{U}): \bar{\partial} F \sim_{\Sigma} 0\right\} \\
\mathcal{G}_{\bullet}^{s} & : \mathbb{C} \supset \mathcal{U} \mapsto \mathcal{G}_{\bullet}^{s}(\mathcal{U})=\left\{F \in \mathrm{G}^{s}(\mathcal{U}): F \sim_{\Sigma} 0\right\}
\end{aligned}
$$

A existência de funções cortes Gevrey diferenciáveis segundo $M$ equivale, via [Br, Cap.II:Prop.9.2 e Ex.12], ao feixe $\mathrm{G}_{\mathrm{M}}^{s}$ ser soft ou, via isomorfismo (1.18), ao feixe quociente $\mathcal{G}_{\star}^{s} / \mathcal{G}$ s ser C-soft sobre $\Sigma$, onde C denota a família dos compactos de $\mathbb{C}$. Por fim, através de [Br, Cap.II:Prop.15.1], tal existência equivale ao feixe $\mathcal{G}_{*}^{s} / \mathfrak{G}^{s}$ ser $\mathrm{C}$-acíclico sobre abertos de $\mathbb{C}$ ou, equivalentemente,

$$
H_{\mathrm{C}}^{1}\left(\mathcal{U} ; \mathfrak{G}_{\star}^{s} / \mathcal{G}_{\mathfrak{s}} \mid \mathcal{U}\right)=0, \forall \mathcal{U} \subset \mathbb{C} .
$$


Os feixes $\mathcal{G}^{s}$ e $\mathcal{G}_{\bullet}^{s}$ são claramente C-soft, já que a restrição de suas seções aos compactos de $\mathrm{C}$ é sobrejetora. Como o quociente de feixes soft também é soft ([Br, Cap.II:Prop.9.7]), temos a resolução soft

$$
0 \rightarrow \mathcal{G}_{*}^{s} / \mathcal{G}_{\bullet}^{s} \longrightarrow \mathcal{G}^{s} / \mathcal{G}_{\bullet}^{s} \stackrel{\bar{\partial}}{\longrightarrow} \mathcal{G}^{s} / \mathcal{G}_{\bullet}^{s} \rightarrow 0
$$

que permite calcular $H_{\mathrm{C}}^{1}\left(\mathcal{U} ; \mathcal{G}_{\star}^{s} / \mathcal{G}_{\bullet} \mid \mathcal{U}\right)$ através da sequência associada

$$
0 \rightarrow \mathcal{G}_{\star}^{s(\mathcal{U})} / \mathcal{G}_{\bullet}^{s}(\mathcal{U}) \longrightarrow \mathcal{G}^{s}(\mathcal{U}) / \mathcal{G}^{s}(\mathcal{U}) \stackrel{\bar{\partial}}{\longrightarrow} \mathcal{G}^{s}(\mathcal{U}) / \mathcal{G}^{s}(\mathcal{U}) \rightarrow 0
$$

já que

$$
\left.H_{\mathrm{C}}^{1}\left(\mathcal{U} ; \mathcal{G}_{*}^{s} / \mathcal{G}_{\bullet}^{s} \mid \mathcal{U}\right) \simeq{\left(\mathcal{G}^{s}(\mathcal{U}) / \mathcal{G}_{\bullet}^{s}(\mathcal{U})\right.}\right) / \operatorname{Im} \bar{\partial} .
$$

Concluímos então que a existência de funções cortes nas classes $\mathrm{G}_{\mathrm{M}}^{s}(V)$ equivale à validade do seguinte problema em abertos $\mathcal{U}$ de $\mathbb{C}$ :

- dada $F \in \mathcal{G}^{s}(\mathbb{C})$ suportada em $\mathcal{U}$ existe $G \in \mathcal{G}^{s}(\mathbb{C})$ também suportada em $\mathcal{U}$ tal que $(\bar{\partial} F-G) \sim_{\Sigma} 0$.

Finalizamos observando que a condição de $\Sigma$ ser Gevrey, além de suficiente, deve ser necessária para a resolução Gevrey $\bar{\partial}$-flat sobre $\Sigma$ do problema em questão.

\subsection{A questão da diferenciabilidade Gevrey parcial segundo $(M, L)$}

Num primeiro momento o conceito de diferenciabilidade Gevrey parcial segundo $(M, L)$ foi estabelecido a partir das estimativas

$$
\forall \ell \exists C_{\ell} \exists h_{\ell}: \sup _{\bar{U}}\left|\mathrm{M}^{\alpha} L^{\gamma} f\right| \leq C_{\ell} h_{\ell}^{|\alpha|}|\alpha| !^{s}, \forall(\alpha, \gamma) \in \mathbb{Z}_{+}^{m+n},|\gamma| \leq \ell .
$$

Observe que tais estimativas são mais abrangentes que (3.4) e também mais abrangentes que (2.10), já que

$$
(|\alpha|+|\gamma|) !^{s} \leq C_{|\gamma|} h_{|\gamma|}^{|\alpha|}|\alpha| !^{s} .
$$

Com efeito, as classes $\mathrm{G}_{\mathrm{ML}}^{s}(U)$ estavam contidas nas classes $\mathcal{G}_{\mathrm{ML}}^{s}(U)$. 
Infelizmente a técnica utilizada na demonstração do teorema central não se casa com as classes $\mathcal{G}_{\mathrm{ML}}^{s}(U)$ definidas a partir de (5.1), pois a dependência qualquer de $h$ em relação a $\ell$ inviabiliza o Lema 8 . Neste caso é possível mostrar que, para cada $\ell$ fixado, existe $P_{\ell}(\mathrm{M})$ na classe de Kaneko de ordem $s \operatorname{com} P_{\ell}(\mathrm{M}) f$ infinitamente diferenciável em $x$ e de classe $C^{\ell}$ em $t$. Assumindo uma resolubilidade do tipo $C^{\ell}$ em $t$ e utilizando um processo do tipo Mittag Leffler, acreditamos ser possível num futuro próximo enunciar nosso teorema nas classes Gevrey parciais segundo (M, L) definidas a partir de (5.1).

\subsection{A questão da resolubilidade ultradiferen- cial}

Apesar desta tese tratar somente da resolubilidade ultradiferencial relacionada à sequência de Gevrey $\left\{p !^{s}\right\}$, conjecturamos ser possível estender nossos resultados para casos mais gerais de ultradiferenciabilidade, envolvendo sequências $\left\{N_{p}\right\}$ satisfazendo propriedades de

- convexidade logarítmica

$$
N_{p}^{2} \leq N_{p-1} N_{p+1} \quad, \forall p=1,2, \ldots,
$$

- estabilidade segundo operadores ultradiferenciais

$$
N_{p} \leq C H^{p} \min _{0 \leq q \leq p} N_{q} N_{p-q} \quad, \forall p=0,1, \ldots,
$$

- não-quase-analiticidade forte

$$
\sum_{q=p+1}^{\infty} \frac{N_{q-1}}{N_{q}} \leq C p \frac{N_{p-1}}{N_{p}}, \forall p=1,2, \ldots .
$$




\section{Simbologia}

- as variáveis $x=\left(x_{1}, \ldots, x_{m}\right) \in \mathbb{R}^{m}, t=\left(t_{1}, \ldots, t_{n}\right) \in \mathbb{R}^{n}, z=\left(z_{1}, \ldots, z_{m}\right) \in \mathbb{C}^{m}$

- os domínios

$V \ni x, \mathcal{U} \ni z, U \ni(x, t), \mathcal{W} \ni(z, t)$

- os espaços de funções Gevrey

- Gevrey usual

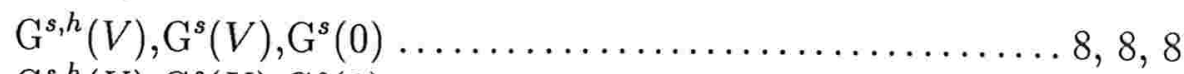

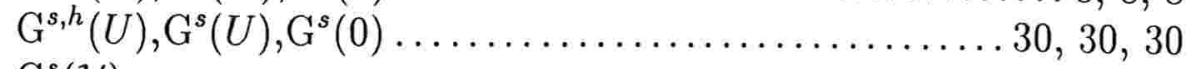
$\mathrm{G}^{s}(\mathcal{U})$

- Gevrey segundo a estrutura hipo-analítica $\mathrm{G}_{\mathrm{M}}^{s, h}(V), \mathrm{G}_{\mathrm{M}}^{s}(V), \mathrm{G}_{\mathrm{M}}^{s}(0)$ $\mathrm{G}_{\mathrm{ML}}^{s, h}(U), \mathrm{G}_{\mathrm{ML}}^{s}(U), \mathrm{G}_{\mathrm{ML}}^{s}(0)$.

- Gevrey hipo-analítico $\mathrm{G}_{\mathrm{ha}}^{s}(U), \mathrm{G}_{\mathrm{ha}}^{s}(0)$ 30,30

- Gevrey parcial segundo a estrutura hipo-analítica $\mathcal{G}_{\mathrm{ML}}^{s}(U), \mathcal{G}_{\mathrm{ML}}^{s}(0)$. 39,39

- Caracterizações Gevrey em $\mathbb{C}^{m}$

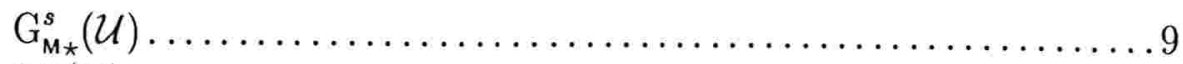

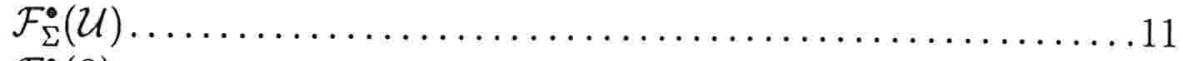

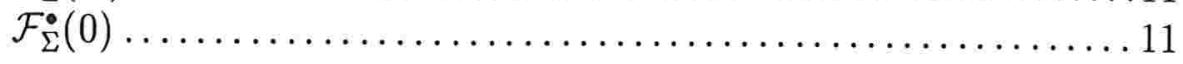

- Caracterizações Gevrey em $\mathbb{C}^{m} \times \mathbb{R}^{n}$

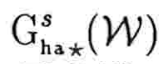




\section{Referências Bibliográficas}

[B] J. Bruna, An extension theorem of Whitney type for non quasianalytic classes of functions. J. LONDON Math. Soc. (2), 22(1980), 495-505

[Bo] Bourbaki, N., Topological Vector Spaces (cap. 1-5). SPRINGERVERLAG, 1987

[Br] Bredon, Glen E., Sheaf Theory. McGraw-Hill BooK ComPANY, 1967

[C] P. Cordaro, Representation of hyperfunction solutions in a hypoanalytic structure. MATH.Z. (2000), a aparecer

[CH1] P. Cordaro e J. Hounie, On local solvability of underdetermined system of vector fields. AMER.J.MATH., 112(1990), 243-270

[CH2] P. Cordaro e J. Hounie, Local solvability for top degree forms in a class of systems of vector fields. AMER.J.Math., 121(1999), 487-495

[CT] P. Cordaro e F. Treves, Homology and cohomology in hypo-analytic structures of the hypersurface type. J.GEOMETRIC ANALYSIS, 1(1991), 39-70

[ChT] S. Chanillo e F. Treves, Local exactness in a class of differential complexes. J.Amer.Math.Soc., 10(2)(1997), 393-426

[D] B. Drost, Holomorphic approximations of ultradifferentiable functions. Math.AnN., 257(1981), 293-316 
[GP] T. Gramchev e P. Popivanov, Partial differential equations: approximate solutions in scales of functional spaces. MATHEMATICAL ReseARCH, 108, Wiley-VCH, Berlin, 2000

[H] L. Hörmander, Linear Partial Differential Operators. SPRINGERVERLAG, 1969

[H1] L. Hörmander, The Analysis of Linear Partial Differential Operators I. SpRInger-Verlag, 1983

[K] H. Komatsu, Ultradistributions I. Structures theorems and a caracterization. J.FAC.ScI.UnIV.Tokio, Sec IA 20(1973), 25-105

[Ka] A. Kaneko, Representation of hyperfunctions by measures and some of its applications. J.FAC.SCI.Univ.TOKIO, Sec IA 19(1972), 321-352

[MT] G. Mendoza e F. Treves, Local solvability in a class of overdetermined sysytems of linear PDE. DukE MATH.J., 62(2)(1991), 355-377

[R] L. Rodino, Linear Partial Differential Operators in Gevrey Spaces. World Scientific, 1993

[T] F. Treves, Hypo-analytic structures (local theory). PRInCETON University Press, Princeton, NJ, 1992

[T1] F. Treves, On the local integrability and local solvability of systems of vector fields. ACTA MATH., 151(1983),1-48

[T2] F. Treves, Integral Representation of Solutions of First-Order Linear Partial Differential Equations. ANN. SC. NORM. SUP. PISA, Serie IV Vol. II 1(1976),1-35 